\title{
ESTABILIDADE DE EQUAÇÕES DIFERENCIAIS RETARDADAS ATRAVÉS \\ DO MÉTODO DAS FUNÇÕES DICOTÔMICAS
}

\author{
Maria Aparecida Bená
}

Orientador: Prof. Dr. Luiz Antonio Vieira de Carvalho

Tese apresentada ao Instituto de Ciências Matemáticas de São Carlos, da Universidade de São Paulo, para obtenção do Título de Doutor em Ciências - Área : Matemática (Análise).

São Carlos - SP

1993 


\section{AGRADECIMENTOS}

- A todos aqueles que direta ou indiretamente contribuiram para a realização deste trabalho.

Em especial:

- Ao PROF.DR.LUIZ ANTONIO VIEIRA DE CARVALHO, pela confiança, orientação e paciência com que sempre nos atendeu.

- Ao PROF.DR.JOSÉ GERALDO DOS REIS, com quem discutimos várias partes deste trabalho, pela amizade, incentivo e valiosas sugestões apresentadas.

- Aos MEUS PAIS, pelo amor e pela força interior.

- A minha irmã SOLANGE e ao meu cunhado CARLOS ALEXANDRE, pelo carinho e apoio.

- Aos amigos JAIR, SÍLVIA, LÍDIA e SOLANGE, pela convivência, pelo carinho e incentivo, valiosos nos momentos mais difíceis.

- Aos PROFESSORES e AMIGOS do DGFM da FFCLRP-USP, pela oportunidade concedida. 
- A SANDRA e ao GODOY, pelas acolhidas carinhosas, amizade e pelo incentivo nos momentos importantes.

- A ELIZABETH L.MORETTI E SILVA, a LAURA AP. D.RUY TURI e a SONIA R.DERIGI BORGES, funcionárias da área acadêmica do ICMSC-USP, pela eficiência, amizade e pelo tratamento carinhoso.

- $\dot{A}$ CAPES, pela concessão da bolsa de estudo.

- A SONIA AP. NALI DE PAULA e ao APARECIDO DONISETI DA COSTA, pela dedicação e capricho com que digitaram este trabalho. 
Aos meus pais e a Solange 


\begin{abstract}
This work is devoted to the study of the stability of Retarded Differential Equations using Dichotomic Maps.

Firstly, some concepts about Dichotomic Maps and the theorems for stability and asymptotic stability are established. Several applications of this method are also made. We call attention to the equation $x^{\prime}(t)=-\lambda x(t)+\lambda f(x(t-1))$ that has been widely used in many fields.

The power of the method can be appreciated by the improvements of many results and by the simplicity of the "functionals" that are employed. In this context, the study of the equation $x^{\prime}(t)=-b(t) x(t-r)$ shows to be a nice example. In this study, in spite of dropping some restrictive conditions, we still could determine a large region of the stability in terms of the parameters.

Some results about solutions that go to zero in an oscillatory way and about equations with infinite delays are also obtained.
\end{abstract}




\section{RESUMO}

Este trabalho é dedicado ao estudo da estabilidade de Equações Diferenciais Retardadas usando Funções Dicotômicas.

Inicialmente, alguns conceitos sobre Função Dicotômica e os teoremas para estabilidade e estabilidade assintótica são estabelecidos. Várias aplicações deste método também são feitas. Chamamos a atenção para a equação $x^{\prime}(t)=-\lambda x(t)+\lambda f(x(t-1))$ que tem sido amplamente usada em muitos campos.

A força do método pode ser apreciada pelo aperfeiçoamento de muitos resultados e pela simplicidade dos "funcionais" empregados. Nesse sentido, um bom exemplo é dado pela equação $x^{\prime}(t)=-b(t) x(t-r)$. Apesar de eliminarmos várias hipóteses restritivas no estudo da referida equação, conseguimos, ainda assim, ampliar a região de estabilidade em termos dos parâmetros.

Alguns resultados sobre soluções que tendem a zero de maneira oscilatória e sobre equações com retardamento infinito são obtidos. 


\section{ÍNDICE}

INTRODUÇÃO

CAPÍTULO 1 - Fatos Básicos e o Conceito de Função Dicotômica .01

CAPÍTULO 2 - Teoremas Fundamentais para Estabilidade e Estabilidade Assintótica usando Funções Dicotômicas ................08

CAPÍTUlO 3 - Aplicações da Teoria das Funções Dicotômicas no Estudo de Algumas Equações Diferenciajs Retardadas

CAPÍTULO 4 - Generalização de Alguns Resultados de Lakshmikantham e Leela através de Funções Dicotômicas .37

CAPÍTULO 5 - Análise da Estabilidade de Equações com Retardamento Variável com o Tempo ........................... 56 


\section{INTRODUÇÃO}

O problema de determinar-se a estabilidade do equilíbrio de uma equação diferencial foi sempre um problema de grande interesse, mesmo no caso de soluções de equações diferenciais ordinárias, isto porque é muito difícil determinar-se a forma exata de uma solução geral. Com o surgimento das equações com retardamento, este fato tornou-se mais agudo, pois aumentaram sensivelmente as dificuldades da determinação de soluções. Em razão deste fato, os métodos que determinam a estabilidade de um equilíbrio sem conhecimento explícito da solução, tornaram-se ferramentas importantíssimas. Um exemplo marcante neste sentido é o chamado Segundo Método ou Método Direto de Liapunov, que por sua vasta aplicação dispensa maiores comentários. Veja, por exemplo, [8], [10], [13], [16], [17], [20].

Um passo a frente nesta direção foi dado pelos Teoremas Tipo Razumikhin [13], [16], que permitem a determinação de estabilidade em certos casos não abrangidos por Funcionais de Liapunov.

O Método das Funções Dicotômicas estabelecido por Carvalho e Ferreira [5] para equações a diferenças finitas, e posteriormente por Carvalho e Cooke [4] para equações diferenciais diferenças é mais um avanço nesta linha. O objetivo do presente trabalho é fazer uma extensão deste método para equações retardadas não autônomas. Sua característica básica neste caso, é a não exigência de que a "derivada" do funcional ao longo das soluções seja sempre negativa. Podemos ter a derivada positiva em um instante $t_{1}$, desde que exista um instante $t_{2}, t_{2}<t_{1}$, tal que $o$ valor do funcional no instante $t_{2}$ seja maior ou igual do que seu valor no instante $t_{1}$, no caso de estabilidade simples, ou maior do que uma função deste valor no caso da estabilidade assintótica. Estas exigências são comuns também nos teoremas do tipo Razumikhin. Entretanto, quando se usam estes teoremas para se determinar a estabilidade assintótica, exigese que a "derivada" além de menor do que zero, seja majorada por uma função com propriedades específicas. A determinação desta função não é, em geral, um problema 
trivial.

Já no caso das Funções Dicotômicas, a existência desta função não é necessária. Estes conceitos serão precisamente definidos no corpo do trabalho.

A principal vantagem deste método é que podemos usar funcionais extremamente simples com bons resultados. No que observamos, parece-nos que o funcional $V(x)=x^{2}$ resolve a maioria dos problemas.

Vamos estudar especificamente equações não autônomas do tipo

$$
\begin{aligned}
x^{\prime}(t) & =f\left(t, x_{t}\right), \quad t \geq t_{0} \\
x_{t_{0}} & =\varphi
\end{aligned}
$$

e também apresentar várias aplicações significativas em equações que têm sido amplamente estudadas, conseguindo em alguns casos melhorar resultados existentes na literatura e, além disso, obtê-los de uma forma mais simples.

Um dos aspectos que devemos salientar é que, embora Funcionais de Liapunov e mesmo os Teoremas tipo Razumikhin apresentem certas dificuldades quando existem soluções que tendem a um equilíbrio de forma oscilatória, este fato geralmente parece não apresentar grandes problemas com o uso do Método das Funções Dicotômicas.

Este trabalho é composto de cinco capitulos. O primeiro capítulo, como seu próprio título indica, restringe-se à apresentação de elementos básicos necessários para atingirmos nossos objetivos. No segundo capítulo, estabelecemos os teoremas que garantem estabilidade simples e assintótica usando Funções Dicotômicas. Gostaríamos de ressaltar que os Lemas (2.2) e (2.3) facilitam muito a demonstração do Teorema (2.4) tornando-o bastante compreensível. Queremos destacar também as comparações feitas entre o Método das Funções Dicotômicas e os Teoremas de Razumikhin que são apresentadas no final deste capítulo.

O terceiro capitulo é destinado a aplicações do Método das Funções Dicotômicas a algumas classes particulares de equações diferenciais retardadas. São estudadas quatro 
classes de equações, em que condições para a estabilidade e a estabilidade assintótica das mesmas são estabelecidas. Ressaltamos aqui a Aplicação 4, ou seja, apresentamos um estudo da equação

$$
\begin{aligned}
x^{\prime}(t) & =-\lambda x(t)+\lambda f(x(t-1)) \\
x_{0} & =\varphi
\end{aligned}
$$

que tem sido amplamente abordada em virtude de suas aplicações em diversas áreas da ciência, tais como: Ótica, Ecologia, Fisiologia, Epidemiologia.

O quarto capítulo foi motivado pelo trabalho de Lakshmikantham e Leela [18]. Neste trabalho, os autores estudaram basicamente a estabilidade da equação:

$$
x^{\prime}(t)=-a \int_{t-\tau}^{t}\left[x(\tau)+x^{3}(\tau)\right] d \tau
$$

O estudo é feito através de Funções de Liapunov e de técnicas de comparação entre soluções de equações diferenciais ordinárias e equações diferenciais retardadas. Usando Funções Dicotômicas, obtivemos os mesmos resultados de uma maneira bem simples e além disso, conseguimos estendê-los para uma classe de equações da qual a equação anterior é um caso particular. Também são apresentados alguns resultados sobre equações do tipo:

$$
x^{\prime}(t)=-b g(x(t))-a \int_{t-r}^{t} g(x(\tau)) d \tau
$$

Finalmente, no quinto e último capítulo, estudamos algumas equações com retardamento variável, isto é, equações da forma 


$$
x^{\prime}(t)=-b x(t-r(t))
$$

onde $r:[0, \infty) \rightarrow R_{+}$é uma função contínua.

Queremos destacar neste capítulo as equações, que embora com retardamento constante, têm um comportamento bastante semelhante ao de equações com retardamento variável, isto porque a variação do retardo é substituída pela variação do parâmetro $b$, que ao invés de ser constante, passa a ser uma função contínua do tempo. Neste caso particular, ressaltamos a equação:

$$
x^{\prime}(t)=-b(t) x(t-r)
$$

Em [3], Burton e Hatvani demonstraram, impondo várias hipóteses sobre a função $b$, que as soluções desta equação tendem a zero. Nós conseguimos, com hipóteses menos restritivas, mostrar também este fato, além da estabilidade assintótica do equilíbrio nulo, o que não é feito pelos referidos autores. Em [3] é também exigido que a função $b$ seja periódica de período $r$, o que não usamos, e assim expandimos a classe de funções para as quais estes resultados são válidos.

Finalmente, estabelecemos, através do Método das Funções Dicotômicas, a estabilidade de uma equação com retardamento infinito. 


\section{Capítulo 1}

\section{FATOS BÁSICOS E O CONCEITO DE FUNÇÃO DICOTÔMICA}

A fim de estabelecermos formalmente o contexto em que vamos trabalhar, serão consideradas equações diferenciais funcionais retardadas não autônomas do tipo

$$
\begin{aligned}
x^{\prime}(t) & =f\left(t, x_{t}\right), \quad t \geq t_{0} \\
x_{t_{0}} & =\varphi
\end{aligned}
$$

onde $f: R \times C \rightarrow R^{n}$ é uma função contínua, leva conjuntos limitados em conjuntos limitados, $f(t, 0)=0, \varphi \in C=C\left([-r, 0], R^{n}\right), r>0 ; C$ indica o espaço de Banach das aplicações contínuas $\varphi$ de $[-r, 0]$ em $R^{n} \operatorname{com}$ a norma $\|\varphi\|=\sup \{|\varphi(\theta)|: \theta \in[-r, 0]\}$, onde $|$.$| denota uma norma do R^{n}$.

Ao longo de todo este trabalho a função $f$ satisfaz condições suficientes para garantir a existência, unicidade e dependência contínua das soluções de (1.1) em relação às condições iniciais.

Dada uma função contínua $x:\left[t_{0}-r, t_{0}+\tau\right) \rightarrow R^{n}, \tau>0$, definimos, para $t \in\left[t_{0}, t_{0}+\tau\right)$, a função $x_{t}:[-r, 0] \rightarrow R^{n}$ da seguinte forma: $x_{t}(\theta)=x(t+\theta)$, $\theta \in[-r, 0]$.

Assim se denotarmos por $x\left(., t_{0}, \varphi\right)$ a solução de $(1.1)$ tal que $x_{t_{0}}\left(., t_{0}, \varphi\right)=\varphi$, $x_{t}\left(., t_{0}, \varphi\right)$ tem um significado claro como elemento de $C$, ou seja, $x_{t}\left(., t_{0}, \varphi\right)(\theta)=$ $=x\left(t+\theta, t_{0}, \varphi\right), \theta \in[-r, 0]$.

Note que a solução através de $\varphi=0$ indicada por $x\left(., t_{0}, 0\right)$ é a solução nula, a qual é também chamada de "equilíbrio nulo". 
Maiores informações sobre a teoria geral das Equações Diferenciais Funcionais Retardadas podem ser encontradas nas referências seguintes: [8], [10], [12], [13].

Observação: $O$ símbolo $\square$ será usado para indicar o final das demonstrações de lemas e teoremas.

A seguir vamos apresentar algumas definições e notações que serão necessárias no decorrer deste trabalho.

Definição 1.1: A solução nula de (1.1) é dita estável se dados $t_{0} \in R$ e $\epsilon>0$, existir $\delta=\delta\left(\epsilon, t_{0}\right)>0$, tal que se $\|\varphi\|<\delta$ então $\left\|x_{t}\left(., t_{0}, \varphi\right)\right\|<\epsilon$, para $t \geq t_{0}$.

Definição 1.2: A solução nula de (1.1) é dita assintoticamente estável se for estável e além disso existir $\gamma=\gamma\left(t_{0}\right)>0$ tal que se $\|\varphi\|<\gamma$ então $x\left(t, t_{0}, \varphi\right) \rightarrow 0$ quando $t \rightarrow \infty$.

Definição 1.3: A solução nula de (1.1) é dita uniformemente estável se o $\delta \mathrm{da}$ definição (1.1) for independente de $t_{0}$.

Definição 1.4: A solução nula de (1.1) é dita uniformemente assintoticamente estável se for uniformemente estável e existir $\delta>0$ tal que para cada $\epsilon>0$, existe um $T(\epsilon)$ tal que se $\|\varphi\|<\delta$ então $\left\|x_{t}\left(., t_{0}, \varphi\right)\right\|<\epsilon$, para todo $t \geq t_{0}+T(\epsilon)$.

É fato conhecido da teoria de Equações Diferenciais Funcionais [6], [13], [16], que se a equação (1.1) for autônoma ou periódica, os conceitos de estabilidade assintótica e estabilidade assintótica uniforme são equivalentes.

Várias relações entre esses conceitos para Equações Diferenciais Ordinárias podem 
ser encontrados em [7] e para Equações Retardadas, em [14].

Definição 1.5: Um conjunto compacto $A \subset C$, invariante pelo fluxo definido por $f$, é dito um atrator global da equação (1.1) se para toda $\varphi \in C, d\left(x_{t}\left(., t_{0}, \varphi\right), A\right) \rightarrow 0$, quando $t \rightarrow \infty$.

Observação: No caso em que o conjunto A se reduz a um ponto de equilíbrio, ou seja, uma função constante, o fato deste ponto ser um atrator global não implica em que o mesmo seja estável.

Definição 1.6: Seja $U \subset R^{n}$ um conjunto e $0 \in U$. Uma função contínua $V: R^{n} \rightarrow R$ é dita definida positiva em $U$ se $V(0)=0$ e $V(x)>0$ para $x \neq 0, x \in U$.

Definição 1.7: Uma função $V: R \times R^{n} \rightarrow R$ é dita definida positiva em $U, 0 \in U$, se $V$ é continua, $V(t, 0)=0$ para qualquer $t \in R$ e existe uma função $\alpha: R \rightarrow R$ contínua, estritamente crescente em $[0, \infty)$, com $\alpha(0)=0$ tal que $V(t, x) \geq \alpha(|x|)$, para $(t, x) \in R \times U$.

Definição 1.8: Seja $V: R \times R^{n} \rightarrow R$ uma função contínua. Definimos a "derivada de $V$ ao longo das soluções de (1.1), $V^{\prime}(t, \varphi) ”$, por

$$
V^{\prime}(t, \varphi)=\lim _{h \rightarrow 0^{+}} \sup \frac{1}{h}[V(t+h, x(t+h, t, \varphi))-V(t, x(t, t, \varphi))]
$$

Dados:

(i) uma função diferenciável $V$, satisfazendo $V(t, 0)=0$, 
(ii) uma vizinhança $\Omega$ da origem em $C$,

(iii) $t, T \in R \operatorname{com} T \geq 0, t \geq t_{0}+T$,

(iv) $p: R_{+} \rightarrow R_{+}$uma função contínua, não decrescente, $p(y)>y, \quad y \in(0, \delta)$, para algum $\delta>0$ fixado,

passamos a definir os seguintes subconjuntos de $C$ :

$$
\begin{aligned}
& \Omega_{+}^{\prime}(t, T)=\left\{\psi \in \Omega: V^{\prime}\left(t+T, x_{t+T}(., t, \psi)\right)>0\right\}, \\
& \Omega_{0}^{\prime}(t, T)=\left\{\psi \in \Omega: V^{\prime}\left(t+T, x_{t+T}(., t, \psi)\right)=0\right\}, \\
& \Omega_{-}(s, t, T)=\{\psi \in \Omega: V(t+T, x(t+T, t, \psi))-V(t+s, x(t+s, t, \psi))<0\}, \\
& s \in[-r, T) \text {, } \\
& \Omega_{0}(s, t, T)=\{\psi \in \Omega: V(t+T, x(t+T, t, \psi))-V(t+s, x(t+s, t, \psi))=0\} \text {, } \\
& s \in[-r, T) \text {, } \\
& \Omega_{-}^{p}(s, t, T)=\{\psi \in \Omega: p(V(t+T, x(t+T, t, \psi)))-V(t+s, x(t+s, t, \psi))<0\}, \\
& s \in[-r, T) \text {. }
\end{aligned}
$$

Dado um conjunto $A, A^{*}$ representa o conjunto $A \backslash\{0\}, \bar{A}$, o fecho de $\mathrm{A}$ e $\Omega_{-}^{0}(s, t, T)=\Omega_{-}(s, t, T) \cup \Omega_{0}(s, t, T)$. 
Definição 1.9: Dizemos que uma função diferenciável $V: R \times R^{n} \rightarrow R$ é dicotômica relativamente à equação (1.1) se para cada par $\left(t_{0}, t\right), t \geq t_{0}$, existirem um $T=T\left(t_{0}, t\right)$, $0 \leq T\left(t_{0}, t\right) \leq t-t_{0}$ e uma vizinhança $\Omega$ da origem em $C$, tal que

$$
\left(\bar{\Omega}_{+}^{\prime}(t-T, T) \bigcup \Omega_{0}^{\prime}(t-T, T)\right) \subset \bigcup_{s} \Omega_{-}^{0}(s, t-T, T), \quad-r \leq s<T
$$

Definição 1.10: Se $V$ for dicotômica, e além disso existir um $M, 0<M<\infty$ e uma função $p$ satisfazendo (iv) tal que:

$$
\begin{gathered}
T=T\left(t_{0}, t\right) \leq M, \quad \text { para todo } t \geq t_{0}, \quad e \\
\left(\bar{\Omega}_{+}^{\prime}(t-T, T) \bigcup \Omega_{0}^{\prime}(t-T, T)\right)^{*} \subset \bigcup_{s} \Omega_{-}^{p}(s, t-T, T), \quad-r \leq s<T,
\end{gathered}
$$

dizemos então que $V$ é estritamente dicotômica relativamente à equação (1.1).

Observação: $\mathrm{Na}$ definição de dicotomia estrita, a inclusão $\left(\bar{\Omega}_{+}^{\prime}(t-T, T) \cup \Omega_{0}^{\prime}(t-T, T)\right)^{*} \subset \bigcup_{s} \Omega_{-}^{p}(s, t-T, T)$ implica que $\Omega_{0}^{\prime}(t-T, T) \cap \Omega_{0}(s, t-T, T)=\{0\}$, para todo $s \in[-r, T)$, ou seja, o único elemento de $C$ existente nesta interseç̧ão é a função $\psi \equiv 0$.

Formalmente temos o seguinte resultado:

Lema 1.1: Suponhamos $V$ estritamente dicotômica relativamente à equação (1.1), satisfazendo $V(t, 0)=0$. Então $\Omega_{0}^{\prime}(t-T, T) \cap \Omega_{0}(s, t-T, T)=\{0\}$, para todo $s$, $-r \leq s<T$. Isto significa que a única solução tal que o funcional $V$ tem derivada nula num ponto $t$ e é constante em todo intervalo $[t-T-r, t]$ é a solução nula. 
Demonstração: Suponhamos, por absurdo, que exista $\psi \neq 0$, $\psi \in \Omega_{0}^{\prime}(t-T, T) \cap \Omega_{0}(s, t-T, T)$. Então $\psi \in \Omega$ é tal que $V^{\prime}\left(t, x_{t}(., t-T, \psi)\right)=0 \quad e \quad V(t, x(t, t-T, \psi))=V(t-T+s, x(t-T+s, t-T, \psi))$ para todo $s \in[-r, T)$. Como $V$ é estritamente dicotômica em relação à equação (1.1), segue que

$$
V\left(t-T+s_{0}, x\left(t-T+s_{0}, t-T, \psi\right)\right)>p(V(t, x(t, t-T, \psi))),
$$

para algum $s_{0} \in[-r, T)$.

Assim, pela característica da função $p$ podemos escrever:

$$
V(t, x(t, t-T, \psi))<p(V(t, x(t, t-T, \psi)))<V\left(t-T+s_{0}, x\left(t-T+s_{0}, t-T, \psi\right)\right)
$$

para algum $s_{0} \in[-r, T)$, o que contraria nossa suposição inicial. Portanto,

$$
\Omega_{0}^{\prime}(t-T, T) \bigcap \Omega_{0}(s, t-T, T)=\{0\}, \quad \text { para todo } s,-r \leq s<T .
$$

Observação: Comparando-se as definições de dicotomia e dicotomia estrita estabelecidas neste trabalho com as correspondentes definições para o caso autônomo dadas em [4], nota-se que: a Definição (1.9) é uma extensão da definição de dicotomia estabelecida em [4], e portanto são semelhantes. Com relação à dicotomia estrita o mesmo não ocorre. Exige-se na Definição (1.10), a existência de uma função $p$ com certas propriedades especificas. Este fato implica que $\Omega_{0}^{\prime}(t-T, T) \cap \Omega_{0}(s, t-T, T)=\{0\}$, como mostra o Lema (1.1). No caso autônomo, como é natural, as condições exigidas são menos restritivas, pois pede-se apenas que a interseç̧ão dos conjuntos seja a função nula, não havendo necessidade da existência da função $p$.

As figuras (1) e (2) ajudam a esclarecer os significados das Definições (1.9) e (1.10) respectivamente. 


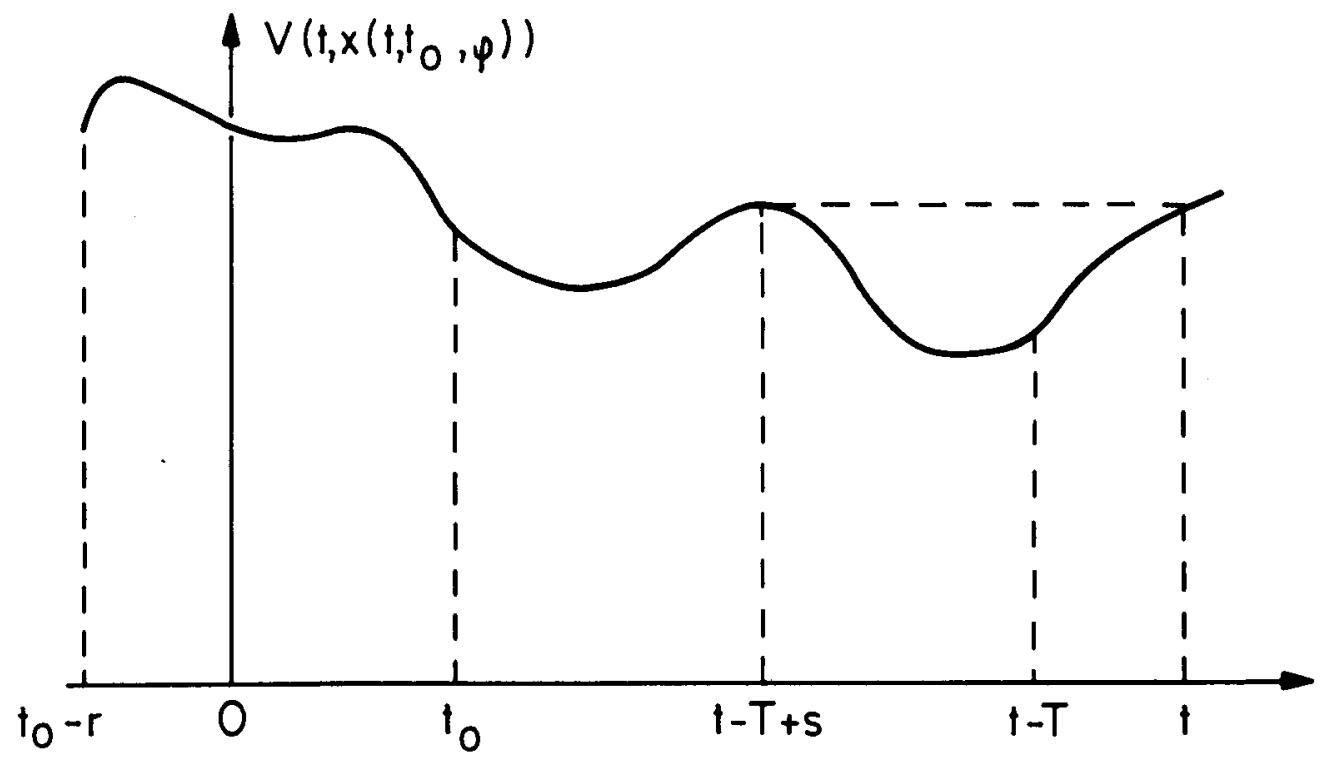

Figura 1 - Dicotomia

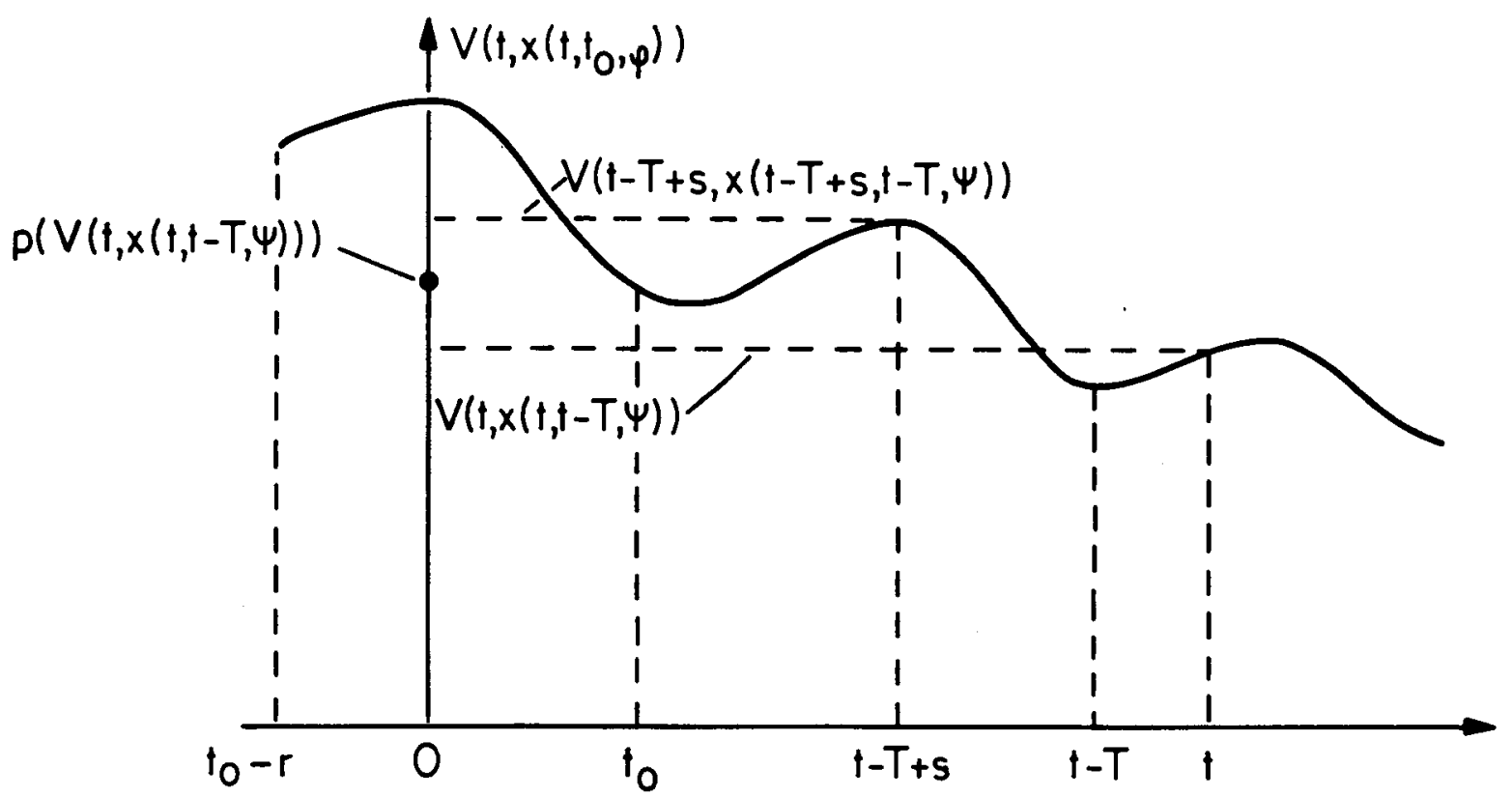

Figura 2 - Dicotomia estrita 


\section{Capítulo 2}

\section{TEOREMAS FUNDAMENTAIS PARA ESTABILIDADE E ESTABILIDADE ASSINTÓTICA USANDO FUNÇÕES DICOTÔMIC̣AS}

Neste capítulo, enunciamos e demonstramos os teoremas que garantem estabilidade e estabilidade assintótica da solução nula da equação (1.1), utilizando a noção de Função Dicotômica. Para atingir este objetivo, necessitamos de dois lemas auxiliares.

No final do capítulo, apresentamos uma comparação entre o uso da teoria das Funções Dicotômicas e os teoremas tipo Razumikhin no estudo da estabilidade de equações do tipo (1.1).

Exibimos também um exemplo em Equações Diferenciais Ordinárias para mostrar que o Teorema (2.4) não pode ser melhorado.

Teorema 2.1: Suponhamos que $u, v: R_{+} \rightarrow R_{+}$sejam funções contínuas, não decrescentes com $u(0)=v(0)=0, u(x)$ e $v(x)$ positivas para $x>0$.

Se existir uma função $V: R \times R^{n} \rightarrow R$ definida positiva, dicotômica relativamente à equação (1.1), satisfazendo

$$
u(|x|) \leq V(t, x) \leq v(|x|), \quad t \in R, \quad x \in R^{n}
$$

então o equilíbrio nulo de (1.1) é estável.

Demonstração: Para qualquer $\epsilon>0$, existe um $\delta_{0}=\delta_{0}(\epsilon), 0<\delta_{0}<\epsilon$ tal que $v\left(\delta_{0}\right)<u(\epsilon)$. 
Dado $t_{0} \in R$, a continuidade de $x\left(t, t_{0}, \varphi\right)$ com relação a $\varphi$, a continuidade de $V$ e o fato de $V(t, 0)=0$ garantem a existência de um $\delta_{1}=\delta_{1}\left(\epsilon, t_{0}\right)>0$ tal que se $\|\varphi\|<\delta_{1}$ então $V\left(t, x\left(t, t_{0}, \varphi\right)\right)<u(\epsilon)$ para $t \in\left[t_{0}-r, t_{0}+\tau\right]$, para algum $\tau>0$ suficientemente pequeno.

Seja $\delta=\min \left\{\delta_{0}, \delta_{1}\right\}$. Mostremos que se $\|\varphi\|<\delta$ então $V\left(t, x\left(t, t_{0}, \varphi\right)\right)<u(\epsilon)$, para $t \geq t_{0}$. Suponhamos, por absurdo, que exista um primeiro $t_{1}>t_{0}+\tau$ tal que

$$
\begin{gathered}
V\left(t_{1}, x\left(t_{1}, t_{0}, \varphi\right)\right)=u(\epsilon) \quad e \\
V\left(t, x\left(t, t_{0}, \varphi\right)\right)<u(\epsilon) \quad \text { para } t<t_{1} .
\end{gathered}
$$

Isto implica que $V^{\prime}\left(t_{1}, x_{t_{1}}\left(., t_{0}, \varphi\right)\right) \geq 0$. Assim, como $V$ é dicotômica relativamente à equação (1.1), existe um $T=T\left(t_{0}, t_{1}\right), 0 \leq T\left(t_{0}, t_{1}\right) \leq t_{1}-t_{0}$ e uma vizinhança $\Omega$ da origem em $C$ tal que

$$
x_{t_{1}-T}\left(., t_{0}, \varphi\right) \in \bar{\Omega}_{+}^{\prime}\left(t_{1}-T, T\right) \subset \bigcup_{s} \Omega_{-}^{0}\left(s, t_{1}-T, T\right), \quad-r \leq s<T
$$

Segue que $x_{t_{1}-T}\left(., t_{0}, \varphi\right) \in \Omega_{-}^{0}\left(s, t_{1}-T, T\right)$, para algum $s \in[-r, T)$, isto é,

$$
V\left(t_{1}, x\left(t_{1}, t_{1}-T, x_{t_{1}-T}\left(., t_{0}, \varphi\right)\right)\right)-V\left(t_{1}+s-T, x\left(t_{1}+s-T, t_{1}-T, x_{t_{1}-T}\left(., t_{0}, \varphi\right)\right)\right) \leq 0
$$

ou seja,

$$
V\left(t_{1}, x\left(t_{1}, t_{0}, \varphi\right)\right) \leq V\left(t_{1}-\bar{s}, x\left(t_{1}-\bar{s}, t_{0}, \varphi\right)\right)
$$

para algum $\bar{s} \in(0, T+r]$. Portanto $V\left(t_{1}, x\left(t_{1}, t_{0}, \varphi\right)\right)<u(\epsilon)$, em contradição com nossa suposição inicial. 
Logo, se $\|\varphi\|<\delta$ então $V\left(t, x\left(t, t_{0}, \varphi\right)\right)<u(\epsilon)$, para $t \geq t_{0}$.

Por hipótese, temos que

$$
u\left(\left|x\left(t, t_{0}, \varphi\right)\right|\right) \leq V\left(t, x\left(t, t_{0}, \varphi\right)\right)<u(\epsilon), \text { para } t \geq t_{0}
$$

Como $u$ é não decrescente, segue que $\left|x\left(t, t_{0}, \varphi\right)\right|<\epsilon$, para $t \geq t_{0}$. Portanto o equilíbrio nulo de (1.1) é estável.

Lema 2.2: Sejam $u, v$ e $V$ como no Teorema (2.1). Além disso, suponhamos que $V$ seja estritamente dicotômica relativamente à equação (1.1). Então, sempre que para um determinado $t_{1}, t_{1} \geq t_{0}$ tivermos $V\left(t_{1}, x\left(t_{1}, t_{0}, \varphi\right)\right)>c$ e $V^{\prime}\left(t_{1}, x_{t_{1}}\left(., t_{0}, \varphi\right)\right) \geq 0$, existirá um $\rho=\rho(c)>0$ tal que

$$
V\left(t_{1}, x\left(t_{1}, t_{0}, \varphi\right)\right)-V\left(t_{1}-T+s, x\left(t_{1}-T+s, t_{0}, \varphi\right)\right)<-\rho
$$

para algum $s \in[-r, T)$ onde $T$ é como na Definição (1.10).

Demonstração: Pelo Teorema (2.1), existem $\delta_{1}>0$ e $\delta_{2}>0$ tal que se $\|\varphi\|<\delta_{1}$ então $V\left(t, x\left(t, t_{0}, \varphi\right)\right)<\delta_{2}, \quad$ para $t \geq t_{0}$.

Se $V\left(t_{1}, x\left(t_{1}, t_{0}, \varphi\right)\right)>c$, segue que $c<V\left(t_{1}, x\left(t_{1}, t_{0}, \varphi\right)\right)<\delta_{2}$.

Se $V^{\prime}\left(t_{1}, x_{t_{1}}\left(., t_{0}, \varphi\right)\right) \geq 0$, de acordo com a definição de dicotomia estrita, existem $T=T\left(t_{0}, t_{1}\right)$ e $0<M<\infty, 0 \leq T\left(t_{0}, t_{1}\right) \leq M$, tal que

$$
x_{t_{1}-T}\left(., t_{0}, \varphi\right) \in\left(\bar{\Omega}_{+}^{\prime}\left(t_{1}-T, T\right) \bigcup \Omega_{0}^{\prime}\left(t_{1}-T, T\right)\right)^{*} \subset \bigcup_{s} \Omega_{-}^{p}\left(s, t_{1}-T, T\right)
$$

$s \in[-r, T)$, isto é, 
$p\left(V\left(t_{1}, x\left(t_{1}, t_{1}-T, x_{t_{1}-T}\left(., t_{0}, \varphi\right)\right)\right)<V\left(t_{1}-T+s, x\left(t_{1}-T+s, t_{1}-T, x_{t_{1}-T}\left(., t_{0}, \varphi\right)\right)\right)\right.$,

para algum $s \in[-r, T)$, ou seja,

$$
p\left(V\left(t_{1}, x\left(t_{1}, t_{0}, \varphi\right)\right)\right)<V\left(t_{1}-T+s, x\left(t_{1}-T+s, t_{0}, \varphi\right)\right)
$$

para algum $s \in[-r, T)$.

Seja $0<\rho(c)<\min \left\{p(y)-y, y \in\left[c, \delta_{2}\right]\right\}$. Assim, segue que

$$
V\left(t_{1}-T+s, x\left(t_{1}-T+s, t_{0}, \varphi\right)\right)>p\left(V\left(t_{1}, x\left(t_{1}, t_{0}, \varphi\right)\right)\right)>V\left(t_{1}, x\left(t_{1}, t_{0}, \varphi\right)\right)+\rho(c) .
$$

Portanto,

$$
V\left(t_{1}, x\left(t_{1}, t_{0}, \varphi\right)\right)-V\left(t_{1}-T+s, x\left(t_{1}-T+s, t_{0}, \varphi\right)\right)<-\rho(c),
$$

para algum $s \in[-r, T)$ e $\quad 0 \leq T=T\left(t_{0}, t_{1}\right) \leq M$.

Lema 2.3: Suponhamos $V$ de classe $C^{1}$ e estritamente dicotômica relativamente à equação (1.1). Seja $r>0$ um retardamento finito e $0<M<\infty$ a constante que aparece na Definição (1.10). Então para cada $t_{1} \in R, t_{1} \geq t_{0}$ em que $V^{\prime}\left(t_{1}, x_{t_{1}}\left(., t_{0}, \varphi\right)\right)>0$, existe um ponto $t_{2} \in\left[t_{1}, t_{1}+M+r\right]$ tal que $V^{\prime}\left(t_{2}, x_{t_{2}}\left(., t_{0}, \varphi\right)\right)=0$ e $t_{2}$ é um ponto de máximo local de $V\left(t, x\left(t, t_{0}, \varphi\right)\right)$.

Demonstração: Seja $t_{1} \in R, t_{1} \geq t_{0} \operatorname{com} V^{\prime}\left(t_{1}, x_{t_{1}}\left(., t_{0}, \varphi\right)\right)>0$. Suponhamos, por absurdo, que $V^{\prime}\left(t, x_{t}\left(., t_{0}, \varphi\right)\right) \geq 0$ para $t \in\left[t_{1}, t_{1}+M+r\right]$. Como 


$$
V\left(t_{1}+M+r, x\left(t_{1}+M+r, t_{0}, \varphi\right)\right)=V\left(t, x\left(t, t_{0}, \varphi\right)\right)+\int_{t}^{t_{1}+M+r} V^{\prime}\left(u, x_{u}\left(., t_{0}, \varphi\right)\right) d u
$$

para $t \in\left[t_{1}, t_{1}+M+r\right]$, se não existisse ponto de máximo neste intervalo, teríamos

$$
V\left(t_{1}+M+r, x\left(t_{1}+M+r, t_{0}, \varphi\right)\right) \geq V\left(t, x\left(t, t_{0}, \varphi\right)\right)
$$

$t \in\left[t_{1}, t_{1}+M+r\right]$. Logo em $t_{1}+M+r$ teríamos $V^{\prime}\left(t_{1}+M+r, x_{t_{1+M+r}}\left(., t_{0}, \varphi\right)\right) \geq 0 \mathrm{e}$ $V\left(t_{1}+M+r, x\left(t_{1}+M+r, t_{0}, \varphi\right)\right) \geq V\left(t, x\left(t, t_{0}, \varphi\right)\right)$, para $t \in\left[t_{1}, t_{1}+M+r\right]$. Assim,

$$
p\left(V\left(t_{1}+M+r, x\left(t_{1}+M+r, t_{0}, \varphi\right)\right)\right)>V\left(t, x\left(t, t_{0}, \varphi\right)\right), \quad t \in\left[t_{1}, t_{1}+M+r\right]
$$

em contradição com o fato de $V$ ser estritamente dicotômica.

Teorema 2.4: Suponhamos que $V$ satisfaça todas as hipóteses do Teorema (2.1), e além disso, que $V$ seja de classe $C^{1}$ e estritamente dicotômica com relação à equação (1.1). Então o equilíbrio nulo de (1.1) é assintoticamente estável.

Demonstração: Sejam $\delta$ e $u(\epsilon)$ como no Teorema (2.1). Tomemos $\gamma \leq \delta$ e mostremos que se $\|\varphi\|<\gamma$ então $x\left(t, t_{0}, \varphi\right) \rightarrow 0$, quanto $t \rightarrow \infty$.

Para simplificar a notação, denotemos $x\left(t, t_{0}, \varphi\right)$ por " $x(t)$ " e $x_{t}\left(., t_{0}, \varphi\right)$ por " $x_{t}$ ".

Com relação a $x_{t}$ existem duas alternativas: existe um $\bar{t}$ tal que $V^{\prime}\left(t, x_{t}\right) \leq 0$ para todo $t \geq \bar{t}$ ou se isto não ocorrer, o Lema (2.3) garante a existência de uma sequência crescente $\left(m_{n}\right), m_{n} \rightarrow \infty$, quando $n \rightarrow \infty$ tal que $V^{\prime}\left(m_{n}, x_{m_{n}}\right)=0$ e $V\left(m_{n}, x\left(m_{n}\right)\right)$ é máximo local.

Notemos que pela dicotomia estrita, para cada $m_{n}, n \in N$, existe um $T\left(m_{n}\right)$ tal que $x_{m_{n}-T\left(m_{n}\right)} \in \bar{\Omega}_{+}^{\prime}\left(m_{n}-T\left(m_{n}\right), T\left(m_{n}\right)\right)$. 
Vamos tratar inicialmente o caso em que existe tal sequência $\left(m_{n}\right)$.

Podemos definir uma nova sequência $\left(t_{n}\right)$, subsequência de $\left(m_{n}\right)$, da seguinte forma: seja $t_{1}=m_{1} ; t_{2}$ é o primeiro elemento da sequência $\left(m_{n}\right)$, digamos $m_{n_{0}}$, tal que $m_{n_{0}}-t_{1} \geq M+r$. De maneira geral, definimos $t_{k}, k \geq 2$ como o primeiro elemento da sequência $\left(m_{n}\right)$, digamos $m_{n_{j}}$ tal que $m_{n_{j}}-t_{k-1} \geq M+r$. A Figura (3) ilustra como a subsequência $\left(t_{n}\right)$ é construída.

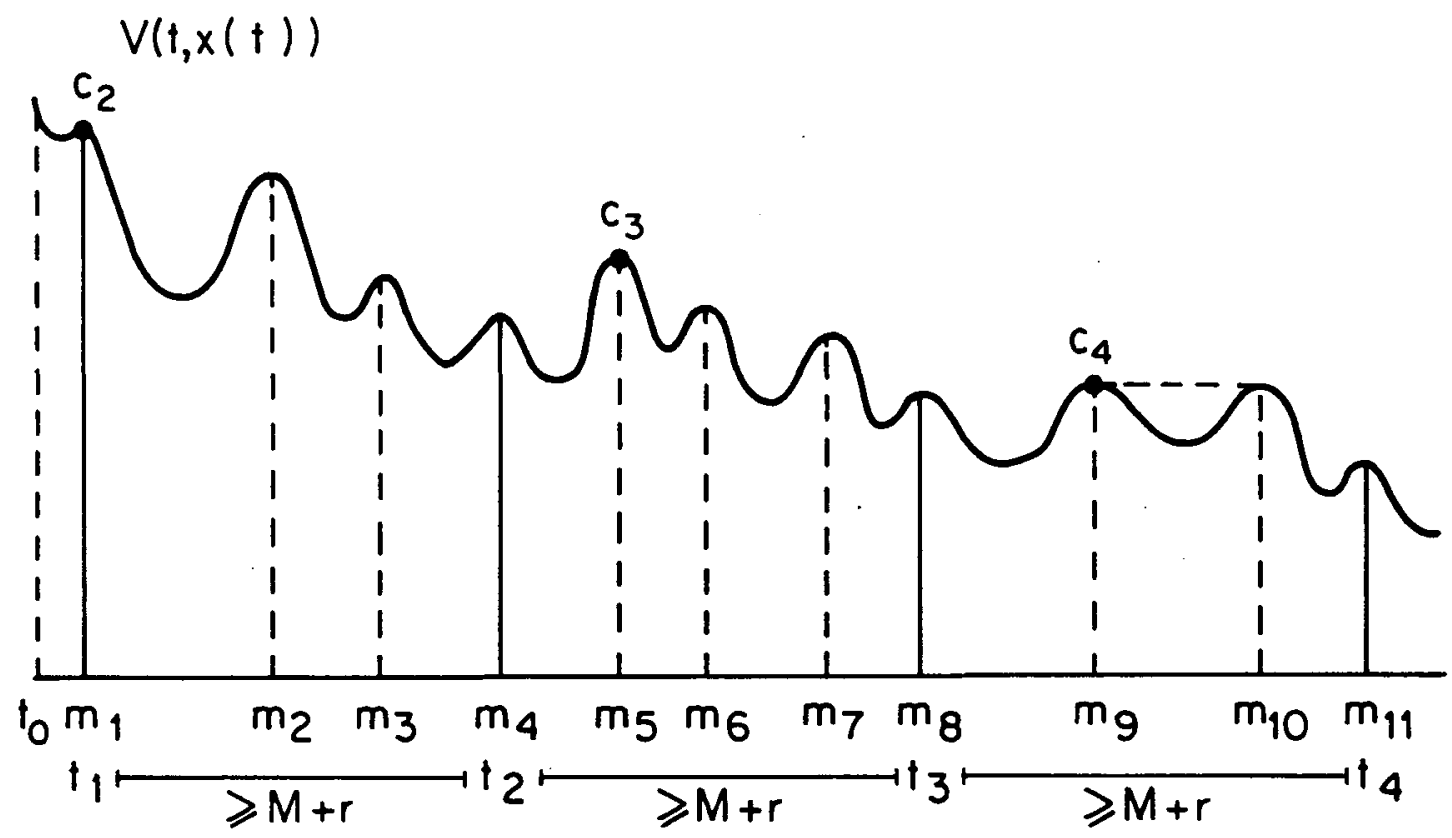

Figura 3

Note que é sempre possível construir a sequência $\left(t_{n}\right)$, pois caso contrário teríamos um $t_{k_{0}}$ fixado tal que $m_{n}<t_{k_{0}}+M+r$, para todo $n \in N$, contra a hipótese que $m_{n} \rightarrow \infty$, quando $n \rightarrow \infty$.

Sejam

$$
\begin{gathered}
c_{k}=\operatorname{máx}\left\{V(t, x(t)): t_{k-1} \leq t \leq t_{k}\right\}, \quad k=2,3, \ldots \quad e \\
\ell_{k}=\min \left\{t \in\left[t_{k-1}, t_{k}\right]: c_{k}=V\left(\ell_{k}, x\left(\ell_{k}\right)\right)\right\}, \quad k=2,3, \ldots
\end{gathered}
$$


Se $\ell_{k} \in\left(t_{k-1}, t_{k}\right)$ então $V^{\prime}\left(\ell_{k}, x_{\ell_{k}}\right)=0$ e também, por construção, $V^{\prime}\left(t_{k}, x_{t_{k}}\right)=0$. Isto implica que existe um $T\left(\ell_{k}\right)$ tal que $x_{\ell_{k}-T\left(\ell_{k}\right)} \in \bar{\Omega}_{+}^{\prime}\left(\ell_{k}-T\left(\ell_{k}\right), T\left(\ell_{k}\right)\right)$, para $k \geq 2$. Como $V$ é estritamente dicotomica, segue que

$$
p\left(V\left(\ell_{k}, x\left(\ell_{k}\right)\right)\right)<V\left(\ell_{k}-T\left(\ell_{k}\right)+s, x\left(\ell_{k}-T\left(\ell_{k}\right)+s\right)\right)
$$

para algum $s \in\left[-r, T\left(\ell_{k}\right)\right)$.

Seja $s_{0}$ um valor de $s$ para o qual a desigualdade acima se verifica. Então $\ell_{k}-T\left(\ell_{k}\right)+s_{0} \in\left[t_{k-2}, t_{k-1}\right]$, uma vez que o mesmo não pode pertencer ao intervalo $\left[t_{k-1}, t_{k}\right]$, pois $V\left(\ell_{k}, x\left(\ell_{k}\right)\right)$ é o máximo neste intervalo, e também não pode pertencer a intervalos anteriores a $\left[t_{k-2}, t_{k-1}\right]$ pois o comprimento deste intervalo é, por construção, maior que $M+r$ e $M+r>T\left(\ell_{k}\right)+r$. Logo,

$$
V\left(\ell_{k}-T\left(\ell_{k}\right)+s_{0}, x\left(\ell_{k}-T\left(\ell_{k}\right)+s_{0}\right)\right)<V\left(\ell_{k-1}, x\left(\ell_{k-1}\right)\right)=c_{k-1} .
$$

Segue que

$$
p\left(c_{k}\right)<V\left(\ell_{k}-T\left(\ell_{k}\right)+s_{0}, x\left(\ell_{k}-T\left(\ell_{k}\right)+s_{0}\right)\right)<c_{k-1}, \quad k \geq 2 .
$$

Portanto,

$$
c_{k-1}>p\left(c_{k}\right)>c_{k}, \quad k \geq 2
$$


Assim $\left(c_{k}\right)$ é uma sequência estritamente decrescente. Como é limitada, então $\left(c_{k}\right)$ é convergente.

Provemos que $c_{k} \rightarrow 0$, quando $k \rightarrow \infty$. Suponhamos, por absurdo, que $c_{k} \rightarrow c$ quando $k \rightarrow \infty$.

Temos que $V\left(\ell_{k}, x\left(\ell_{k}\right)\right)>c$ e $V^{\prime}\left(\ell_{k}, x_{\ell_{k}}\right)=0$. Como $V$ é estritamente dicotômica, pelo Lema (2.2), existe uma constante $\rho=\rho(c)>0$ tal que

$$
\begin{aligned}
& c_{k}<c_{k-1}-\rho, \quad k \geq 3 \\
& c_{k+1}<c_{k}-\rho<c_{k-1}-2 \rho, \quad k \geq 3
\end{aligned}
$$

Uma aplicação sucessiva deste processo leva-nos à seguinte desigualdade:

$$
c_{k+j}<c_{k}-j \rho, \quad j \in N
$$

Logo $c_{k+j} \rightarrow-\infty$, quando $j \rightarrow \infty$, e assim $c_{k} \rightarrow-\infty$, quando $k \rightarrow \infty$, o que contradiz a nossa suposição. Portanto $c=0$. Segue que $u(|x(t)|) \leq V(t, x(t)) \rightarrow 0$, quando $t \rightarrow \infty$. Usando as hipóteses sobre a função $u$, obtemos que $x(t) \rightarrow 0$, quando $t \rightarrow \infty$.

Analisemos agora o caso em que existe um $\bar{t}$ tal que $V^{\prime}\left(t, x_{t}\right) \leq 0$ para $t \geq \bar{t}$. Construimos a sequência $\left(t_{n}\right)$ como anteriormente até obter um $n_{0}$ tal que $t_{n_{0}}=\bar{t}$ ou $t_{n_{0}}+M+r \geq \bar{t}$.

Como $V^{\prime}\left(t, x_{t}\right) \leq 0$ para $t \geq \bar{t}$, segue que $V(t, x(t))$ é decrescente para $t \geq \bar{t}$.

Da limitação de $V$, segue que $V(t, x(t)) \rightarrow c$ quanto $t \rightarrow \infty$. Assim,

$$
V(t, x(t))-V(t-M+s, x(t-M+s)) \rightarrow 0
$$


quando $t \rightarrow \infty$, para todo $s \in[-r, M), M$ da definição de dicotomia estrita. Logo, $x_{t} \rightarrow \Omega_{0}(s, t-M, M)$, quando $t \rightarrow \infty$, para todo $s \in[-r, M)$.

Por hipótese, $V^{\prime}\left(t, x_{t}\right) \leq 0$ para $t \geq \bar{t}$. Se existir um $\sigma>0$ tal que $V^{\prime}\left(t, x_{t}\right)<-\sigma$ para $t \geq \bar{t}$, podemos escrever $V(t, x(t))=V(\bar{t}, x(\bar{t}))+\int_{\bar{t}}^{t} V^{\prime}\left(u, x_{u}\right) d u$ e assim $V(t, x(t)) \rightarrow-\infty$, quando $t \rightarrow \infty$, o que é um absurdo. Se tal fato não ocorrer, dada a sequência $(1 / n), n \in N$, existe uma sequência $t_{n}^{\prime} \rightarrow \infty$, quando $n \rightarrow \infty$ tal que $V^{\prime}\left(t_{n}^{\prime}, x_{t_{n}^{\prime}}\right)>-1 / n$. Logo, $x_{t_{n}^{\prime}} \rightarrow \Omega_{0}^{\prime}\left(t_{n}^{\prime}-M, M\right)$, quando $n \rightarrow \infty$. Portanto $x_{t_{n}^{\prime}} \rightarrow \Omega_{0}^{\prime}\left(t_{n}^{\prime}-M, M\right) \cap \Omega_{0}\left(s, t_{n}^{\prime}-M, M\right)$, quando $n \rightarrow \infty$. Pelo Lema (1.1), temos que $x_{t_{n}^{\prime}} \rightarrow 0$, quando $n \rightarrow \infty$. Logo $x\left(t_{n}^{\prime}\right) \rightarrow 0$ quando $n \rightarrow \infty$, e assim $V\left(t_{n}^{\prime}, x\left(t_{n}^{\prime}\right)\right) \rightarrow 0$ quando $n \rightarrow \infty$. Sendo $V$ decrescente, segue que $V(t, x(t)) \rightarrow 0$, quando $t \rightarrow \infty$. Como $u(|x(t)|) \leq V(t, x(t)), u(0)=0$ e $V(t, x(t)) \rightarrow 0$, quando $t \rightarrow \infty$, concluimos que $x(t) \rightarrow 0$, quando $t \rightarrow \infty$ e portanto o equilíbrio nulo de (1.1) é assintoticamente estável.

À primeira vista, pode-se pensar na teoria das funções dicotômicas como um caso particular dos teoremas tipo Razumikhin. Isto não é verdade, e nós faremos algumas considerações nesta linha.

Os teoremas de Razumikhin comparam $V(s, x(s))$ em dois pontos $s=s_{1}$ e $s=s_{2}$, $s_{1}<s_{2}, s_{1}, s_{2} \in[t-r, t+k r]$, onde $k$ é um inteiro não negativo fixado [13,cap.5]. Para funções dicotômicas esta exigência não é necessária.

Este fato leva-nos a concluir que os teoremas tipo Razumikhin são inadequados para o estudo de equações com retardamento infinito.

Entretanto, é no caso da estabilidade assintótica que a diferença entre os dois métodos torna-se mais evidente. Neste caso, mesmo para $T=0$, o método das funções dicotômicas não é um caso particular do teorema de Razumikhin. No teorema de Razumikhin devemos garantir que se $V(t+\theta, \varphi(\theta))<p(V(t, \varphi(0))), \theta \in[-r, 0]$ então $V^{\prime}(t, \varphi) \leq-w(|\varphi(0)|)$, onde $w$ e $p$ são funções contínuas, não decrescentes com $w(y)>0$, para $y>0$ e $p(y)>y$ para $y>0$. 
$\mathrm{Na}$ teoria das funções dicotômicas, para o caso $T=0$, exigimos apenas que $V(t+\theta, \varphi(\theta))>p(V(t, \varphi(0)))$ para algum $\theta \in[-r, 0)$ quando $V^{\prime}(t, \varphi) \geq 0$.

Assim, não necessitamos exibir uma função $w$ com as propriedades acima.

Além disso, se as hipóteses do teorema de Razumikhin estiverem satisfeitas, as hipóteses do teorema das funções dicotômicas também estarão. Portanto, a teoria das funções dicotômicas é menos exigente do que os teoremas tipo Razumikhin e pode ser considerada uma generalização dos mesmos.

Como foi observado no Capítulo 1, os conceitos de estabilidade assintótica e estabilidade assintótica uniforme são equivalentes quando a equação (1.1) for autônoma ou periódica.

Vamos mostrar que o resultado obtido no Teorema (2.4) não pode ser melhorado nem mesmo para Equações Diferenciais Ordinárias, como mostra o seguinte exemplo.

Seja

$$
\begin{aligned}
& x^{\prime}(t)=\frac{-x(t)}{t+1}, \quad t \geq 0 \\
& x\left(t_{0}\right)=x_{0}
\end{aligned}
$$

Consideremos o funcional $V(x)=x^{2} / 2$. Assim,

$$
V^{\prime}\left(x_{t}\right)=x(t) x^{\prime}(t)=-x^{2}(t) /(t+1)
$$

Notemos que $V^{\prime}\left(x_{t}\right)<0$, mas não existe uma função $w$ com as condições exigidas anteriormente tal que $V^{\prime}\left(x_{t}\right) \leq-w(|x(t)|)$ pois $V^{\prime}\left(x_{t}\right) \rightarrow 0$ quando $t \rightarrow \infty$ para $x(t)$ limitado. Como $V^{\prime}\left(x_{t}\right)<0$, o conjunto $\left(\bar{\Omega}_{+}^{\prime}(t-T, T) \cup \Omega_{0}^{\prime}(t-T, T)\right)^{*}=\phi$, 
para todo $t$ e para algum $T \geq 0$. Assim a inclusão $\left(\bar{\Omega}_{+}^{\prime}(t-T, T) \cup \Omega_{0}^{\prime}(t-T, T)\right)^{*} \subset \bigcup_{s} \Omega_{-}^{p}(s, t-T, T), s \in[0, T)$ é trivialmente satisfeita. Portanto $V$ é estritamente dicotômica relativamente à equação (2.2).

Escolhendo $u(x)=x^{2} / 4$ e $v(x)=x^{2}$ para $x>0$, a desigualdade (2.1) é verificada $\mathrm{e}$ pelo Teorema (2.4) a solução nula de (2.2) é assintoticamente estável.

Por outro lado, a solução de $(2.2)$ é dada por $x(t)=x\left(t_{0}\right)\left(t_{0}+1\right) /(t+1)$.

Se existissem $\delta>0$, e para cada $\epsilon>0$ dado, um $T(\epsilon)$ tal que $\left|x_{0}\right|<\delta$ implicasse $|x(t)|<\epsilon$, para $t \geq t_{0}+T(\epsilon)$, teríamos necessariamente

$$
\left(t_{0}+1\right)((\delta / \epsilon)-1)<T(\epsilon), \quad \text { para } \delta>\epsilon
$$

o que implicaria $T(\epsilon) \rightarrow \infty$ quando $t_{0} \rightarrow \infty$, o que mostra que as condições do Teorema (2.4) não implicam estabilidade assintótica uniforme, ou seja, o Teorema (2.4) não pode ser melhorado, nesse sentido. 


\section{Capítulo 3}

\section{APLICAÇÕES DA TEORIA DAS FUNÇÕES DICOTÔMICAS NO ESTUDO DE ALGUMAS EQUAÇÕES DIFERENCIAIS RETARDADAS}

Neste capítulo, por simplicidade de notação, usaremos " $x(t)$ " para denotar a solução $x\left(t, t_{0}, \varphi\right)$.

Aplicação 1: Consideremos a equação

$$
\begin{aligned}
x^{\prime}(t) & =f(x(t)-x(t-1)) \\
x_{0} & =\varphi
\end{aligned}
$$

onde $f: R \rightarrow R$ é contínua, satisfaz $x f(x)<0$, para $x \neq 0$ e $\varphi \in C([-1,0], R)$. Nessas condições, o equilíbrio nulo de (3.1) é estável.

De fato:

Observe que a hipótese $x f(x)<0$ para $x \neq 0$ com $f$ contínua implica em $f(0)=0$. Seja $V(x)=x^{2} / 2$. Assim

$$
V^{\prime}\left(x_{t}\right)=x(t) x^{\prime}(t)=x(t) f(x(t)-x(t-1))
$$


Suponhamos $V^{\prime}\left(x_{t}\right) \geq 0$. Vamos considerar os seguintes casos:

(i) $x(t) \geq 0$

Então $f(x(t)-x(t-1)) \geq 0$. Segue pela hipótese que $(x(t)-x(t-1)) \leq 0$, ou seja, $x(t-1) \geq x(t)$. Portanto $V(x(t-1)) \geq V(x(t))$.

(ii) $x(t) \leq 0$.

Como no caso (i) isto nos leva a $(x(t)-x(t-1)) \geq 0$ ou seja, $x(t) \geq x(t-1)$, o que implica $|x(t-1)| \geq|x(t)|$. Logo $V(x(t-1)) \geq V(x(t))$.

Portanto, em qualquer um dos casos, temos que $V^{\prime}\left(x_{t}\right) \geq 0$ implica em $V(x(t))-V(x(t-1)) \leq 0$. Assim a condição de dicotomia da Definição (1.9) está satisfeita para $T=0$ e $s=-1$.

Escolhendo $u(x)=x^{2} / 4$ e $v(x)=x^{2}$, para $x>0$ no Teorema (2.1), segue que o equilíbrio nulo de (3.1) é estável.

\section{Aplicação 2: Consideremos a equação}

$$
\begin{aligned}
x^{\prime}(t) & =-b(t) f(t, x(t-r)) \\
x_{r} & =\varphi
\end{aligned}
$$

satisfazendo as seguintes condições:

$f(t, x)$ é contínua para $t \geq 0$ e $x \in R$,

$f(t, x)=0$ se, e somente se, $x=0$,

$f$ tem derivadas parciais contínuas, 
$|(\partial f / \partial x)(t, x)|<L, \quad L>0, \quad t \geq 0, \quad x \in R$

$|x|^{2} \leq x f(t, x), \quad t \geq 0, x \in R$,

$b:[0, \infty) \rightarrow(0, \mu) \quad$ é contínua, com $\quad \mu<1 / r L^{2}$

$\varphi \in C([0, r], R)$.

Com essas hipóteses, a solução nula de (3.2) é assintoticamente estável.

De fato: Seja $V(x)=x^{2} / 2$.

Para $t \geq 2 r$, podemos escrever a equação (3.2) como:

$$
\begin{aligned}
x^{\prime}(t) & =-b(t) f(t, x(t))+[b(t) f(t, x(t))-b(t) f(t, x(t-r))]= \\
& =-b(t) f(t, x(t))+b(t) \int_{t-r}^{t}(\partial f / \partial x)(t, x(\theta))(-b(\theta) f(\theta, x(\theta-r))) d \theta .
\end{aligned}
$$

Temos que

$$
\begin{aligned}
V^{\prime}\left(x_{t}\right) & =x(t) x^{\prime}(t)= \\
& =-b(t) x(t) f(t, x(t))+b(t) x(t) \int_{t-r}^{t}(\partial f / \partial x)(t, x(\theta))(-b(\theta) f(\theta, x(\theta-r))) d \theta .
\end{aligned}
$$

Se $V^{\prime}\left(x_{t}\right) \geq 0$, vem que

$$
-b(t) x(t) f(t, x(t))+b(t) x(t) \int_{t-r}^{t}(\partial f / \partial x)(t, x(\theta))(-b(\theta) f(\theta, x(\theta-r))) d \theta \geq 0
$$

Utilizando o Teorema do Valor Médio e as hipóteses dadas inicialmente, esta última integral pode ser majorada da seguinte forma: 
$b(t) x(t) \int_{t-r}^{t}(\partial f / \partial x)(t, x(\theta))(-b(\theta) f(\theta, x(\theta-r))) d \theta \leq$

$\leq b(t)|x(t)| \int_{t-r}^{t}|(\partial f / \partial x)(t, x(\theta))||b(\theta)||f(\theta, x(\theta-r))| d \theta \leq$

$\leq b(t)|x(t)| \int_{t-\tau}^{t} L^{2} b(\theta)|x(\theta-r)| d \theta \leq$

$\leq b(t)|x(t)| \int_{t-\tau}^{t} L^{2} b(\theta)\left\|x_{t-r}\right\| d \theta$.

Sendo $b(t)<\mu<1 / r L^{2}$, podemos tomar A, $0<A<1$ tal que $b(t) r L^{2} \leq A<1$. Assim,

$$
b(t)|x(t)| \int_{t-r}^{t} L^{2} b(\theta)\left\|x_{t-r}\right\| d \theta \leq A b(t)|x(t)|\left\|x_{t-r}\right\| .
$$

Logo, o primeiro membro da desigualdade que aparece em (3.3) pode ser majorado por:

$-b(t) x(t) f(t, x(t))+A b(t)|x(t)|\left\|x_{t-r}\right\| \geq$

$\geq-b(t) x(t) f(t, x(t))+b(t) x(t) \int_{t-r}^{t}(\partial f / \partial x)(t, x(\theta))(-b(\theta) f(\theta, x(\theta-r))) d \theta \geq 0$.

Portanto,

$$
-b(t) x(t) f(t, x(t))+A b(t)|x(t)|\left\|x_{t-r}\right\| \geq 0 .
$$


Usando a hipótese $|x|^{2} \leq x f(t, x)$, segue que $x f(t, x) \geq 0$.

Temos os seguintes casos a considerar:

(i) $x(t) \geq 0$ e $f(t, x(t)) \geq 0$

Como $|x(t)|^{2} \leq x(t) f(t, x(t))$, vem que $x^{2}(t) \leq x(t) f(t, x(t))$ e assim $-x(t) \geq-f(t, x(t))$. Logo, usando este fato em (3.4) podemos escrever:

$-b(t)|x(t)|^{2}+A b(t)|x(t)|\left\|x_{t-r}\right\| \geq$

$\geq-b(t) x(t) f(t, x(t))+A b(t)|x(t)|\left\|x_{t-r}\right\| \geq 0$.

Assim,

$-b(t)|x(t)|^{2}+A b(t)|x(t)|\left\|x_{t-r}\right\| \geq 0, \quad$ ou seja,

$b(t)|x(t)|\left(-|x(t)|+A\left\|x_{t-r}\right\|\right) \geq 0, \quad$ o que implica

$$
\left\|x_{t-r}\right\| \geq \frac{|x(t)|}{A}, \quad 0<A<1
$$

Logo, existe um $B_{1}, \quad 0<A<B_{1}<1$ tal que

$$
\left\|x_{t-r}\right\|>\frac{|x(t)|}{B_{1}}
$$


Assim,

$$
\frac{\left\|x_{t-r}\right\|^{2}}{2}>\frac{|x(t)|^{2}}{2 B_{1}^{2}}, \quad 0<B_{1}<1
$$

Analisemos a seguir o caso

(ii) $x(t) \leq 0$ e $f(t, x(t)) \leq 0$

Como $|x(t)|^{2} \leq x(t) f(t, x(t))$, temos $|x(t)|^{2}+|x(t)| f(t, x(t)) \leq 0$, ou seja, $|x(t)|(|x(t)|+f(t, x(t))) \leq 0$.

Da desigualdade acima devemos ter $f(t, x(t)) \leq-|x(t)|=x(t)$, isto é, $x(t) \geq f(t, x(t))$.

Logo, de maneira análoga ao caso (i) podemos escrever:

$$
b(t)|x(t)|\left(-|x(t)|+A\left\|x_{t-r}\right\|\right) \geq 0
$$

Desta desigualdade, segue a existência de um $B_{2}, \quad 0<A<B_{2}<1$ tal que

$$
\left\|x_{t-r}\right\|>\frac{|x(t)|}{B_{2}}
$$

Portanto,

$$
\frac{\left\|x_{t-r}\right\|^{2}}{2}>\frac{|x(t)|^{2}}{2 B_{2}^{2}}, \quad 0<B_{2}<1
$$


Em ambos os casos analisados, seja $p(y)=y / B^{2}, y>0$, onde $B=\max \left\{B_{1}, B_{2}\right\}$. Assim temos $p(V(x(t)))<V(x(t-T+s))$, para algum $s \in[-r, T)$, onde $T=r$. Logo as condições da Definição (1.10) estão satisfeitas e portanto $V$ é estritamente dicotômica com relação à equação (3.2).

Tomando $u(x)$ e $v(x)$ como na Aplicação 1, segue pelo Teorema (2.4) que o equilíbrio nulo de (3.2) é assintoticamente estável.

Já sabemos pelo Lema (1.1) que $\Omega_{0}^{\prime}(t-r, r) \cap \Omega_{0}(s, t-r, r)=\{0\}$, para todo $s \in[-r, r)$. Entretanto, apenas como ilustração, podemos prová-lo diretamente.

De fato: Seja $\psi \in \Omega_{0}^{\prime}(t-r, r) \cap \Omega_{0}(s, t-r, r)$. Então $\psi \in \Omega$ é tal que:

$$
\begin{gathered}
V^{\prime}(\psi)=-b(t) \psi(0) f(t, \psi(-r))=0 \\
\mathrm{e} \\
\psi^{2}(0) / 2=\psi^{2}(s-r) / 2, \quad \text { para todo } s \in[-r, r) .
\end{gathered}
$$

Pela equação (3.5), segue que $\psi(0)=0$ ou $f(t, \psi(-r))=0$.

A equação (3.6) implica que $\psi$ é constante em $[-r, r)$.

Por hipótese, temos que $f(t, \psi(-r))=0$ se, e somente se, $\psi(-r)=0$.

Se $\psi(0)=0$ ou $\psi(-r)=0$, pelo fato de $\psi$ ser constante, temos necessariamente $\psi \equiv 0$, o que prova o Lema (1.1). 
Um exemplo de uma particular equação onde $f$ e $b$ satisfazem as condições anteriores é a seguinte:

Seja

$$
\begin{aligned}
x^{\prime}(t) & =-b(t) \frac{(a t+2 a)}{(t+1)} x(t-r) \\
x_{r} & =\varphi
\end{aligned}
$$

onde $a \in R, a>1$ e $b:[0, \infty) \rightarrow(0, \mu)$ é uma função contínua, com $\mu<1 / 4 a^{2} r$. Neste caso, $f(t, x)=(a t x+2 a x) /(t+1)$. A função $f$ assim definida satisfaz as hipóteses exigidas na Aplicação 2.

\section{De fato:}

$f(t, x)=0$ se, e somente se, $x=0, f(t, x)$ é contínua para $t \geq 0$ e $x \in R, f$ tem derivadas parciais contínuas com $(\partial f / \partial x)(t, x)$ dada por:

$$
\frac{\partial f}{\partial x}(t, x)=\frac{a t+2 a}{t+1}=g(t)
$$

Note que $g(0)=2 a$ e $g^{\prime}(t)=-a /(t+1)^{2}<0$. Logo $(\partial f / \partial x)(t, x)$ é decrescente em $t$, e para $t \rightarrow \infty, g(t) \rightarrow a$. Portanto, $a<(\partial f / \partial x)(t, x)<2 a$.

Mostremos que: 
se $x \geq 0$, então $f(t, x) \geq x$

se $x \leq 0$, então $f(t, x) \leq x$.

Temos que $a t+2 a>t+1$ pois $a>1$. Então $(a t+2 a) /(t+1)>1$.

Se $x \geq 0$, segue que $x(a t+2 a) /(t+1) \geq x$, ou seja, $f(t, x) \geq x ;$ se $x \leq 0$, $x(a t+2 a) /(t+1) \leq x$. Assim $f(t, x) \leq x$.

Então, como vimos anteriormente, o equilíbrio nulo de (3.7) é assintoticamente estável.

Aplicação 3: A equação abaixo também foi estudada em [25] usando o Método Direto de Liapunov.

Seja

$$
\begin{aligned}
x^{\prime}(t) & =-a(t) x(t)+b(t) x(t-r) \\
x_{0} & =\varphi
\end{aligned}
$$

com $a: R_{+} \rightarrow R_{+} \quad$ e $\quad b: R_{+} \rightarrow R$ contínuas satisfazendo $0<c \leq a(t)<\infty$, $|b(t)| \leq b<\mu c, 0<\mu<1$.

Nessas condições, o equilíbrio nulo de (3.8) é assintoticamente estável.

Para verificar este fato, seja $V(x)=x^{2} / 2$.

Vamos mostrar que $V$ assim definida é estritamente dicotômica com relação à equação (3.8).

Temos $V^{\prime}\left(x_{t}\right)=x(t) x^{\prime}(t)=-a(t) x^{2}(t)+b(t) x(t) x(t-r)$. Se $V^{\prime}\left(x_{t}\right) \geq 0$, segue que 


$$
x(t)(-a(t) x(t)+b(t) x(t-r)) \geq 0 .
$$

Note que $V^{\prime}\left(x_{t}\right) \geq 0$ implica $b(t) \neq 0$.

Para atingirmos nosso objetivo, vamos dividir em dois casos: $x(t) \geq 0$ e $x(t) \leq 0$. Consideremos inicialmente

1) $x(t) \geq 0$

Então devemos ter

$$
\begin{gathered}
-a(t) x(t)+b(t) x(t-r) \geq 0, \quad \text { ou seja, } \\
b(t) x(t-r) \geq a(t) x(t) .
\end{gathered}
$$

Como $a(t)>0$ e $x(t) \geq 0$, vem que $b(t) x(t-r) \geq 0$.

Esta desigualdade pode ocorrer nas seguintes situações:

(i) $b(t)>0$ e $x(t-r) \geq 0$

Assim, utilizando as hipóteses, podemos escrever:

$$
x(t-r) \geq \frac{a(t)}{b(t)} x(t) \geq \frac{c}{b} x(t)>\frac{1}{\mu} x(t)
$$

Logo,

$$
\frac{x^{2}(t-r)}{2}>\frac{x^{2}(t)}{2 \mu^{2}}, \quad 0<\mu<1 .
$$


Escolhendo a função $p$ da forma $p(y)=y / \mu^{2}, y>0$, temos $p(V(x(t)))<V(x(t+s))$, para algum $s \in[-r, 0)$.

(ii) $b(t)<0$ e $x(t-r) \leq 0$

Neste caso, utilizando as hipóteses na desigualdade (3.9) temos

$$
-x(t-r) \geq \frac{a(t)}{-b(t)} x(t) \geq \frac{c}{b} x(t)>\frac{1}{\mu} x(t),
$$

ou seja,

$$
\frac{x^{2}(t-r)}{2}>\frac{x^{2}(t)}{2 \mu^{2}}, \quad 0<\mu<1
$$

Portanto, $p(V(x(t)))<V(x(t+s))$, para algum $s \in[-r, 0)$.

Vejamos agora o segundo caso, ou seja

2) $x(t) \leq 0$

Assim devemos ter

$$
\begin{gathered}
-a(t) x(t)+b(t) x(t-r) \leq 0, \quad \text { ou } \\
b(t) x(t-r) \leq a(t) x(t) .
\end{gathered}
$$


Do fato de $a(t)>0$ e $x(t) \leq 0$, vem que $b(t) x(t-r) \leq 0$.

Temos dois casos:

(i) $b(t)<0$ e $x(t-r) \geq 0$

Nestas condições podemos escrever

$$
x(t-r) \geq \frac{a(t)}{b(t)} x(t)=\frac{a(t)}{-b(t)}(-x(t)) \geq \frac{c}{b}(-x(t))>\frac{1}{\mu}(-x(t)) .
$$

Logo,

$$
\frac{x^{2}(t-r)}{2} \geq \frac{(-x(t))^{2}}{2 \mu^{2}}, \quad 0<\mu<1
$$

Portanto, $p(V(x(t)))<V(x(t+s))$, para algum $s \in[-r, 0)$.

(ii) $\quad b(t)>0$ e $x(t-r) \leq 0$

Segue que

$$
b(t) x(t-r) \leq a(t) x(t)
$$

Como $b(t) \leq b$ e $a(t) \geq c$, vem que $b x(t-r) \leq c x(t)$, isto é, $x(t-r) \leq(c / b) x(t)$. Logo, 


$$
\frac{x^{2}(t-r)}{2} \geq \frac{c^{2}}{b^{2}} \frac{x^{2}(t)}{2}>\frac{1}{\mu^{2}} \frac{x^{2}(t)}{2}, \quad 0<\mu<1
$$

Portanto, $p(V(x(t)))<V(x(t+s))$, para algum $s \in[-r, 0)$.

Assim verificamos que tanto para $x(t) \geq 0$ como para $x(t) \leq 0$ temos: sempre que $V^{\prime}\left(x_{t}\right) \geq 0$, segue que $p(V(x(t)))-V(x(t+s))<0$, para algum $s \in[-r, 0)$.

Observe que, nesta aplicação, o T da Definição (1.10) é dado por $T=0$.

Escolhendo $u(x)$ e $v(x)$ como definidos na Aplicação 1, temos que $V$ é estritamente dicotômica com relação à equação (3.8), e pelo Teorema (2.4) segue que o equilíbrio nulo de (3.8) é assintoticamente estável.

Mais uma vez, como na Aplicação 1, apenas para ilustrar, verifiquemos que $\Omega_{0}^{\prime}(t, 0) \cap \Omega_{0}(s, t, 0)=\{0\}$, para todo $s \in[-r, 0)$.

Seja $\psi \in \Omega_{0}^{\prime}(t, 0) \cap \Omega_{0}(s, t, 0)$. Então $\psi \in \Omega$ e satisfaz

$$
\begin{gathered}
\psi(0)[-a(t) \psi(0)+b(t) \psi(-r)]=0 \\
\mathrm{e} \\
\psi^{2}(0) / 2=\psi^{2}(s) / 2, \text { para todo } s \in[-r, 0) .
\end{gathered}
$$

Por $(3.11) \psi$ é constante em $[-r, 0)$.

A equação (3.10) implica que $\psi(0)=0$ ou $b(t) \psi(-r)=a(t) \psi(0)$. Se $\psi(0)=0$, então $\psi \equiv 0$. Se $b(t) \psi(-r)=a(t) \psi(0)$, consideremos dois casos: $b(t)>0$ ou $b(t)<0$.

Para $b(t)<0$, como $a(t)>0$ e $\psi$ é constante, devemos ter $\psi \equiv 0$. Suponhamos $b(t)>0$ e $\psi \neq 0$. Como $\psi$ é constante temos: 


$$
1=\frac{\psi(-r)}{\psi(0)}=\frac{a(t)}{b(t)} \geq \frac{c}{b}>\frac{1}{\mu}>1
$$

o que é uma contradição.

Logo o caso $b(t)>0$ e $\psi \neq 0$ não ocorre. Portanto, $\psi \equiv 0$.

Aplicação 4: A equação (3.12) abaixo tem sido bastante estudada devido ao fato de ser um importante modelo nas áreas de Ecologia, Ótica e Fisiologia [21], [22], [23], $[24]$.

Seja

$$
\begin{aligned}
x^{\prime}(t) & =-\lambda x(t)+\lambda f(x(t-1)), \lambda>0 \\
x_{0} & =\varphi
\end{aligned}
$$

onde $f: R \rightarrow R$ é contínua e satisfaz a seguinte hipótese:

(H) $f(x) \leq x$ se $x \geq 0, f(x) \geq x$ se $x \leq 0$ e $x f(x)>0$ se $x \neq 0$.

Note que esta condição implica que se $f$ for diferenciável na origem, então $0<f^{\prime}(0) \leq 1$.

Podemos, desta forma, enunciar os seguintes resultados:

a) Se $f$ satisfaz (H), então o equilíbrio nulo de (3.12) é estável.

b) Se além de satisfazer a), $f$ for de classe $C^{1}$ numa vizinhança da origem e $0<f^{\prime}(0)<1$, então o equilíbrio nulo de (3.12) é assintoticamente estável. 
Demonstração de a):

Seja $V(x)=x^{2} / 2$, então

$$
V^{\prime}\left(x_{t}\right)=\lambda x(t)[-x(t)+f(x(t-1))]
$$

Se $V^{\prime}\left(x_{t}\right) \geq 0$, nós podemos supor $x(t) \geq 0$, o que implica em $f(x(t-1)) \geq x(t)$. Da hipótese $(\mathrm{H})$ temos que

$$
x(t-1) \geq f(x(t-1)) \geq x(t)
$$

Assim,

$$
x^{2}(t-1) / 2 \geq x^{2}(t) / 2
$$

isto é, $V(x(t-1)) \geq V(x(t))$.

0 caso $x(t) \leq 0$ é análogo.

Em ambos os casos temos que $V^{\prime}\left(x_{t}\right) \geq 0$ implica em $V(x(t))-V(x(t-1)) \leq 0$. Portanto, a condição de dicotomia da Definição (1.9) está satisfeita para $T=0$ e $s=-1$. 
Escolhemos como anteriormente $u(x)=x^{2} / 4$ e $v(x)=x^{2}, x>0$ e pelo Teorema (2.1) o equilíbrio nulo de (3.12) é estável.

\section{Demonstração de b):}

Como $f \in C^{1}$ e $0<f^{\prime}(0)<1$, existe uma bola $B\left(0, \epsilon_{0}\right)$ tal que para $x \in B\left(0, \epsilon_{0}\right)$, $0<f^{\prime}(x)<1$.

Pela parte a), a origem é estável. Assim existe um $\delta_{0}>0$ tal que $\|\varphi\|<\delta_{0}$ implica $x(t) \in B\left(0, \epsilon_{0}\right)$, para $t \geq 0$. Portanto, para todo $t \geq 0$ e para toda solução $x(t)$, com condição inicial $\varphi$ tal que $\|\varphi\|<\delta_{0}$, segue que $0<f^{\prime}(x(t))<1$.

Se $V^{\prime}\left(x_{t}\right) \geq 0$, temos dois casos a considerar:

19) $x(t) \geq 0$

Então $f(x(t-1)) \geq x(t)$. Pelo Teorema do Valor Médio, podemos escrever:

$$
f(x(t-1))-f(0)=f^{\prime}(c) x(t-1) \geq x(t) .
$$

Como $c \in B\left(0, \epsilon_{0}\right)$, temos $f^{\prime}(c)=K<1$, isto é, $K x(t-1) \geq x(t)$.

Seja D uma constante satisfazendo $0<K<D<1$. Então $x(t-1)>x(t) / D$. Assim,

$$
\frac{x^{2}(t-1)}{2}>\frac{x^{2}(t)}{2 D^{2}}, \quad 0<D<1 .
$$


29) $x(t) \leq 0$

Neste caso, vem que $f(x(t-1)) \leq x(t)$. Pela hipótese $(\mathrm{H})$ temos

$$
x(t) \geq f(x(t-1)) \geq x(t-1) .
$$

Aplicando-se novamente o Teorema do Valor Médio e procedendo de forma análoga ao primeiro caso, segue que

$$
\frac{x^{2}(t-1)}{2}>\frac{x^{2}(t)}{2 D^{2}}, \quad 0<D<1
$$

Em ambos os casos analisados, seja $p(y)=y / D^{2}, \quad y>0$.

Assim temos: sempre que $V^{\prime}\left(x_{t}\right) \geq 0$, a desigualdade $p(V(x(t)))<V(x(t-T+s))$ está satisfeita para $T=0$ e $s=-1$. Então as condições da Definição (1.10) estão satisfeitas, e portanto $V$ é estritamente dicotômica com relação à equação (3.12).

De acordo com o Teorema (2.4), o equilíbrio nulo de (3.12) é assintoticamente estável.

A título de ilustração, vamos verificar diretamente a validade do Lema (1.1), ou seja, mostremos que $\Omega_{0}^{\prime}(t, 0) \cap \Omega_{0}(s, t, 0)=\{0\}$, para todo $s \in[-1,0)$.

Seja $\psi \in \Omega_{0}^{\prime}(t, 0) \cap \Omega_{0}(s, t, 0)$. Então $\psi \in \Omega$ é tal que 


$$
\begin{gathered}
V^{\prime}(\psi)=\lambda \psi(0)[-\psi(0)+f(\psi(-1))]=0 \\
\mathrm{e} \\
\psi^{2}(0) / 2=\psi^{2}(s) / 2, \quad \text { para todo } s \in[-1,0) .
\end{gathered}
$$

Por $(3.13)$ temos $\psi(0)=0$ ou $f(\psi(-1))=\psi(0)$.

Se $\psi(0)=0$, como pela equação $(3.14) \psi$ é constante em $[-1,0)$, segue que $\psi \equiv 0$. Suponhamos que $f(\psi(-1))=\psi(0)$. Como $\psi$ é constante, temos $\psi(-1)=\psi(0)$. Então $\psi(0)=f(\psi(-1))=f(\psi(0))$. Por hipótese, o zero é o único ponto fixo de $f$. Assim $\psi(0)=0$. Logo $\psi \equiv 0$, o que comprova o Lema (1.1). 


\section{Capítulo 4}

\section{GENERALIZAÇÃO DE ALGUNS RESULTADOS DE LAKSHMIKANTHAM E LEELA ATRAVÉS DE FUNÇÕES DICOTÔMICAS}

V. Lakshmikantham e S. Leela estudaram em [18] a equação

$$
x^{\prime}(t)=-b\left[x(t)+x^{3}(t)\right]-a \int_{t-\tau}^{t}\left[x(\tau)+x^{3}(\tau)\right] d \tau
$$

onde $a, b, r$ são constantes positivas.

Este estudo foi feito através de uma combinação do uso de Funções de Liapunov, da teoria de desigualdades diferenciais e dos resultados de comparação entre soluções de equações diferenciais retardadas e equações ordinárias. Estas técnicas são encontradas, por exemplo, em [19].

Através destas técnicas, eles estabeleceram condições nos parâmetros $a, b$ e $r$ para a estabilidade do equilíbrio nulo de (4.1).

Entretanto, como eles próprios ressaltaram, estas técnicas não dão nenhuma informação sobre a estabilidade da solução nula de

$$
x^{\prime}(t)=-a \int_{t-r}^{t}\left[x(\tau)+x^{3}(\tau)\right] d \tau
$$

que é na realidade, o verdadeiro objetivo de [18].

Para resolver este problema, os autores introduziram novos métodos de comparação. 
Usando a teoria das funções dicotômicas, vamos estudar ambas as equações estabelecendo os mesmos resultados, de uma maneira que nos parece mais natural, e ainda mais, estendê-los para uma classe mais ampla de equações diferenciais retardadas, da qual (4.1) e (4.2) são casos particulares.

Mostramos também, que com exigências bastante razoáveis nos parâmetros $a, b$ e $r$ e nas funções envolvidas, os equilíbrios nulos de (4.1) e (4.2) não são apenas estáveis, mas sim, assintoticamente estáveis, resultados que não são estabelecidos em [18].

Vamos inicialmente estudar a estabilidade da equação (4.1).

Suponhamos que $a, b$ e $r$ satisfaçam $a r \leq b$.

Considerando $V(x)=x^{2} / 2$, temos então

$$
V^{\prime}\left(x_{t}\right)=x(t) x^{\prime}(t)=-b x(t)\left[x(t)+x^{3}(t)\right]-a x(t) \int_{t-r}^{t}\left[x(\tau)+x^{3}(\tau)\right] d \tau
$$

O Teorema do Valor Médio para Integrais permite-nos escrever $V^{\prime}\left(x_{t}\right)$ na seguinte forma:

$$
V^{\prime}\left(x_{t}\right)=-b x(t)\left[x(t)+x^{3}(t)\right]-a r x(t)\left[x(u)+x^{3}(u)\right], u \in[t-r, t] .
$$

Quando $V^{\prime}\left(x_{t}\right) \geq 0$, temos:

$$
-b x(t)\left[x(t)+x^{3}(t)\right]-\operatorname{ar} x(t)\left[x(u)+x^{3}(u)\right] \geq 0,
$$

ou seja, 


$$
-\operatorname{ar} x(t)\left[x(u)+x^{3}(u)\right] \geq b x(t)\left[x(t)+x^{3}(t)\right]
$$

$$
(a r / b) x(t)\left[-\left(x(u)+x^{3}(u)\right)\right] \geq x(t)\left[x(t)+x^{3}(t)\right]
$$

\section{Consideremos os seguintes casos:}

(i) $x(t) \geq 0$.

Como $(a r / b) \leq 1$, segue que

$$
-\left[x(u)+x^{3}(u)\right] \geq\left[x(t)+x^{3}(t)\right]
$$

Logo $\left[x(u)+x^{3}(u)\right] \leq 0$ e assim $x(u) \leq 0$. Portanto $|x(u)| \geq x(t)$ e $x^{2}(u) / 2 \geq x^{2}(t) / 2$, o que implica $V(x(u)) \geq V(x(t))$, para algum $u \in[t-r, t]$.

\section{Para o caso}

(ii) $x(t) \leq 0$, temos:

$$
-b\left[x(t)+x^{3}(t)\right]-\operatorname{ar}\left[x(u)+x^{3}(u)\right] \leq 0
$$

ou seja 


$$
-\left[x(t)+x^{3}(t)\right] \leq(a r / b)\left[x(u)+x^{3}(u)\right]
$$

Como $(a r / b) \leq 1$, segue que

$$
-\left[x(t)+x^{3}(t)\right] \leq\left[x(u)+x^{3}(u)\right]
$$

Logo, $\left[x(u)+x^{3}(u)\right] \geq 0$ e assim, $x(u) \geq 0$. Portanto, $-x(t) \leq x(u) \mathrm{e}$ $V(x(u)) \geq V(x(t))$, para algum $u \in[t-r, t]$.

Em ambos os casos temos que $V^{\prime}\left(x_{t}\right) \geq 0$ implica em $V(x(t))-V(x(u)) \leq 0$ para algum $u \in[t-r, t]$. Assim $V$ é dicotômica relativamente à equação (4.1).

Tomando $u(x)=x^{2} / 4$ e $v(x)=x^{2}, x>0$, segue pelo Teorema (2.1) que a solução nula de (4.1) é estável.

Mostremos, agora, que ocorrendo a desigualdade estrita, ou seja $a r<b$, temos estabilidade assintótica da solução nula de (4.1).

Da equação (4.3), vem que

$$
(a r / b) x(t)\left[-\left(x(u)+x^{3}(u)\right)\right] \geq x(t)\left[x(t)+x^{3}(t)\right] .
$$

(i) Como antes, seja $x(t) \geq 0$. Então 


$$
(a r / b)\left[-\left(x(u)+x^{3}(u)\right)\right] \geq\left[x(t)+x^{3}(t)\right] .
$$

Como $(a r / b)<1$, existe um $K>0,(a r / b)<K<1$ tal que

$$
K\left[-\left(x(u)+x^{3}(u)\right)\right]>\left[x(t)+x^{3}(t)\right]
$$

Escolhemos $K_{1}, 0<K<K_{1}<1$ e $K_{1}^{3}>K$.

Assim,

$$
K_{1}^{3}\left[-\left(x(u)+x^{3}(u)\right)\right]>K\left[-\left(x(u)+x^{3}(u)\right)\right]>\left[x(t)+x^{3}(t)\right],
$$

ou seja,

$$
K_{1}^{3}\left[-\left(x(u)+x^{3}(u)\right)\right]>\left[x(t)+x^{3}(t)\right]
$$

o que implica $K_{1}(-x(u))>x(t)$. Se esta última desigualdade não fosse verdadeira, teríamos

$$
K_{1}(-x(u)) \leq x(t)
$$

Logo,

$$
K(-x(u))<K_{1}(-x(u)) \leq x(t), \quad 0<K<K_{1}<1 .
$$


Como $K_{1}^{3}>K$, vem que

$$
K(-x(u))^{3}<K_{1}^{3}(-x(u))^{3} \leq x^{3}(t)
$$

Então $K(-x(u))^{3}<x^{3}(t)$. Segue que

$$
K\left[-\left(x(u)+x^{3}(u)\right)\right]<\left[x(t)+x^{3}(t)\right]
$$

o que é uma contradição.

Logo, a desigualdade $K_{1}(-x(u))>x(t)$ é válida, ou seja,

$$
-x(u)>\frac{1}{K_{1}} x(t), \quad 0<K_{1}<1
$$

\section{Portanto,}

$$
\frac{x^{2}(u)}{2}>\frac{x^{2}(t)}{2 K_{1}^{2}}
$$

Definindo $p(y)=y / K_{1}^{2}, y>0$, temos $V(x(u))>p(V(x(t)))$, para algum $u \in[t-r, t]$.

Para analisar (ii) $x(t) \leq 0$, utilizamos a equação (4.4) e procedemos de forma semelhante ao caso (i). 
Tanto no caso (i) como em (ii) temos: sempre que $V^{\prime}\left(x_{t}\right) \geq 0$, segue que $p(V(x(t)))-V(x(u))<0$, para algum $u \in[t-r, t]$. Assim $V$ é estritamente dicotômica com relação à equação (4.1). Consequentemente o Teorema (2.4) garantenos a estabilidade assintótica da solução nula de (4.1).

Observe, que neste exemplo, $T=0$.

A título de ilustração verifiquemos o Lema (1.1), ou seja mostremos que

$$
\Omega_{0}^{\prime}(t, 0) \bigcap \Omega_{0}(s, t, 0)=\{0\}, \quad \text { para todo } s \in[-r, 0) .
$$

Seja $\psi \in \Omega_{0}^{\prime}(t, 0) \cap \Omega_{0}(s, t, 0)$. Então $\psi \in \Omega$ é tal que

$$
\begin{gathered}
V^{\prime}(\psi)=\psi(0)\left[-b \psi(0)-b \psi^{3}(0)-a \int_{-r}^{0}\left(\psi(\tau)+\psi^{3}(\tau)\right) d \tau\right]=0 \\
\mathrm{e} \\
\psi^{2}(0) / 2=\psi^{2}(s) / 2, \quad \text { para todo } s \in[-r, 0)
\end{gathered}
$$

Pela equação (4.6) $\psi$ é constante em $[-r, 0)$. Por $(4.5) \psi(0)=0$ ou $\left[-b \psi(0)-b \psi^{3}(0)-\right.$ $\left.-a \int_{-r}^{0}\left(\psi(\tau)+\psi^{3}(\tau)\right) d \tau\right]=0$. Se $\psi(0)=0$, como $\psi$ é constante, segue que $\psi \equiv 0$. Se $\left[-b \psi(0)-b \psi^{3}(0)-a \int_{-r}^{0}\left(\psi(\tau)+\psi^{3}(\tau)\right) d \tau\right]=0$, podemos escrever: $\left[-b c-b c^{3}-\right.$ $\left.-\operatorname{ar}\left(c+c^{3}\right)\right]=0$, onde $\mathrm{c}$ é o valor constante assumido por $\psi$. Desta equação vem que $(-b-a r)\left(c+c^{3}\right)=0$, ou seja, $c\left(1+c^{2}\right)=0$, o que implica $c=0$. Portanto $\psi \equiv 0$ e o Lema (1.1) está verificado. 
Vamos, agora, como já ressaltamos, usar a teoria das funções dicotômicas para obter resultados de estabilidade e estabilidade assintótica para uma classe mais geral de equações à qual (4.1) e (4.2) pertencem. Para isso, estabelecemos os seguintes teoremas:

Teorema 4.1: Suponhamos $g: R \rightarrow R$ uma função contínua, não decrescente e impar satisfazendo $x g(x)>0$ para $x \neq 0$.

Sejam $a, b, r$ constantes reais positivas tal que

( $\left.H_{1}\right) \quad a r \leq b$.

Nessas condições, o equilíbrio nulo da equação

$$
x^{\prime}(t)=-b g(x(t))-a \int_{t-r}^{t} g(x(\tau)) d \tau
$$

é estável.

$\left(H_{2}\right)$ Se, além disso, $a r<b$ e $g$ é diferenciável numa vizinhança da origem com $g^{\prime}(x)<M<\infty(M>0)$ nesta vizinhança, segue que o equilíbrio nulo de (4.7) é assintoticamente estável.

Demonstração: Como $g$ é contínua e $x g(x)>0$ para $x \neq 0$, segue que $g(x)=0$ se, e somente se, $x=0$. Logo, $x=0$ é solução de (4.7).

Suponhamos $\left(H_{1}\right)$ satisfeita.

Considerando $V(x)=x^{2} / 2$, vem que:

$$
V^{\prime}\left(x_{t}\right)=x(t) x^{\prime}(t)=-b x(t) g(x(t))-a x(t) \int_{t-r}^{t} g(x(\tau)) d \tau
$$


Aplicando o Teorema do Valor Médio para Integrais, podemos escrever:

$$
V^{\prime}\left(x_{t}\right)=-b x(t) g(x(t))-a r x(t) g(x(u)), \quad u \in[t-r, t]
$$

Se $V^{\prime}\left(x_{t}\right) \geq 0$, segue que

$$
x(t)[-b g(x(t))-\arg (x(u))] \geq 0
$$

Vamos considerar os seguintes casos:

(i) $x(t) \geq 0$

\section{Então}

$$
-b g(x(t))-\arg (x(u)) \geq 0
$$

ou seja,

$$
\begin{gathered}
(-a r / b) g(x(u)) \geq g(x(t)), \\
(a r / b)(-g(x(u))) \geq g(x(t)) .
\end{gathered}
$$


Como $(a r / b) \leq 1$, vem que $-g(x(u)) \geq g(x(t))$, onde $g(x(t)) \geq 0$ pois $g$ é não decrescente e $g(0)=0$. Logo, pelo fato de $g$ ser ímpar, temos $g(-x(u)) \geq g(x(t))$ com $g(-x(u)) \geq 0$. Pelo não decrescimento de $g$, segue que $-x(u) \geq x(t)$ com $x(u) \leq 0$. Portanto, $V(x(u)) \geq V(x(t))$, para algum $u \in[t-r, t]$.

(ii) $x(t) \leq 0$

A técnica de demonstração é semelhante ao caso (i), por isso a omitiremos.

Logo, $V$ é dicotômica relativamente à equação (4.7), pois sempre que $V^{\prime}\left(x_{t}\right) \geq 0$ temos $V(x(t))-V(x(u)) \leq 0$, para algum $u \in[t-r, t]$. Escolhendo $u(x)=x^{2} / 4 \mathrm{e}$ $v(x)=x^{2}, x>0$ no Teorema (2.1), segue que o equilíbrio nulo de (4.7) é estável.

Suponhamos, a seguir, que $\left(\mathrm{H}_{2}\right)$ esteja satisfeita. Mostremos a estabilidade assintótica da solução nula de (4.7).

Consideremos $x(t) \geq 0$. Pela equação (4.8) temos:

$$
\begin{gathered}
(a r / b)(-g(x(u))) \geq g(x(t)), \quad \text { ou seja } \\
g(-x(u)) \geq(b / a r) g(x(t)) .
\end{gathered}
$$

Como $(b / a r)>1$, existe um $A_{1},(b / a r)>A_{1}>1$ tal que

$$
g(-x(u))>A_{1} g(x(t)) .
$$


Esta desigualdade juntamente com o fato de $g$ ter derivada limitada numa vizinhança da origem, implicam que

$$
-x(u)>A_{2} x(t), \quad A_{2}>1
$$

Suponhamos, por absurdo, que tal afirmação seja falsa. Então existe uma sequência $\left(K_{n}\right)$ decrescente, $K_{n}>1, K_{n} \rightarrow 1$ quando $n \rightarrow \infty$ tal que $-x(u) \leq K_{n} x(t)$. Podemos escrever $K_{n}=1+a_{n}, a_{n}>0, a_{n} \rightarrow 0$, quando $n \rightarrow \infty$.

Logo,

$$
-x(u) \leq\left(1+a_{n}\right) x(t)
$$

Por hipótese, $g$ é não decrescente, e assim

$$
g(-x(u)) \leq g\left(x(t)+a_{n} x(t)\right)
$$

Pelo Teorema do Valor Médio, segue que

$$
g\left(x(t)+a_{n} x(t)\right)-g(x(t))=g^{\prime}\left(c_{n}\right) a_{n} x(t), \quad c_{n} \in\left(x(t), x(t)+a_{n} x(t)\right) .
$$

Como $g^{\prime}(x)<M$ podemos escrever: 


$$
g\left(x(t)+a_{n} x(t)\right)<g(x(t))+M a_{n} x(t)
$$

Assim, utilizando essa desigualdade em (4.9) vem que:

$$
g(-x(u)) \leq g\left(x(t)+a_{n} x(t)\right)<g(x(t))+M a_{n} x(t),
$$

ou seja,

$$
g(-x(u))<g(x(t))+M a_{n} x(t)
$$

Passando ao limite para $n \rightarrow \infty$, obtemos $g(-x(u)) \leq g(x(t))$, o que é absurdo, pois $g(-x(u))>g(x(t))$. Logo, $-x(u)>A_{2} x(t), A_{2}>1$. Seja $A_{2}=(1 / B)$, $0<B<1$. Assim, $x^{2}(u) / 2>x^{2}(t) / 2 B^{2}$. Escolhendo $p(y)=y / B^{2}, y>0$, temos $V(x(u))>p(V(x(t)))$, para algum $u \in[t-r, t]$.

Para o caso $x(t) \leq 0$, o procedimento é análogo.

Observe que fazendo $u=t+s$, sempre que $V^{\prime}\left(x_{t}\right) \geq 0$, necessariamente teremos $p(V(x(t)))-V(x(t+s))<0$ para algum $s \in[-r, 0)$. Portanto $V$ é estritamente dicotômica com relação à equação (4.7). Então o equilíbrio nulo de (4.7) é assintoticamente estável de acordo com o Teorema (2.4).

Observação: Se considerarmos a equação: 


$$
x^{\prime}(t)=-b g(x(t))+a \int_{t-r}^{t} g(x(\tau)) d \tau
$$

onde (4.10) é a equação (4.7) com uma alteração de sinal antes da integral, podemos estabelecer o seguinte teorema:

Teorema 4.2: Suponha que $g$ embora não ímpar, satisfaça todas as demais hipóteses do Teorema (4.1). Então:

a) Se $\left(H_{1}\right)$ estiver satisfeita, o equilíbrio nulo de (4.10) é estável.

b) Se $\left(H_{2}\right)$ estiver satisfeita, o equilíbrio nulo de (4.10) é assintoticamente estável.

Demonstração: É análoga à do Teorema (4.1).

\section{Exemplos:}

\section{Exemplo 1: Seja}

$$
x^{\prime}(t)=-b \operatorname{tg}(x(t))-a \int_{t-r}^{t} \operatorname{tg}(x(\tau)) d \tau ; \quad a r<b
$$

A função $g(x)=\operatorname{tg} x$ (função tangente de $x$ ) satisfaz todas as hipóteses do Teorema (4.1). Portanto, a solução nula de (4.11) é assintoticamente estável. 
Exemplo 2: Consideremos

$$
x^{\prime}(t)=-b\left[x(t)+x^{3}(t)\right]-a \int_{t-r}^{t}\left[x(\tau)+x^{3}(\tau)\right] d \tau ; \quad a r<b
$$

Esta equação, que é tratada por Lakshmikantham e Leela em [18], e já abordada por nós em detalhes, é, como afirmamos, um caso particular da equação (4.7). Basta observar que $g(x)=x+x^{3}$ satisfaz todas as exigências do Teorema (4.1). Assim a solução nula de (4.12) é assintoticamente estável.

\section{Exemplo 3:}

$$
x^{\prime}(t)=-b P(x(t))-a \int_{t-\tau}^{t} P(x(\tau)) d \tau ; \quad a r<b
$$

onde $P(x(t))$ é um polinômio com termo independente nulo e todos os expoentes ímpares.Logo, as condições do Teorema (4.1) estão satisfeitas e o equilíbrio nulo de (4.13) é assintoticamente estável.

\section{Exemplo 4:}

$$
x^{\prime}(t)=(b-a r)-b e^{x(t)}+a \int_{t-r}^{t} e^{x(\tau)} d \tau ; \quad a r<b
$$

Esta equação pode ser escrita como

$$
x^{\prime}(t)=-b\left(e^{x(t)}-1\right)+a \int_{t-\tau}^{t}\left(e^{x(\tau)}-1_{1}\right) d \tau .
$$


Basta observar que (4.15) é um caso particular de (4.10), onde $g(x)=e^{x}-1$ é contínua, não decrescente, satisfaz $x g(x)=x e^{x}-x>0$ para $x \neq 0$ e tem derivada limitada numa vizinhança da origem. Assim o equilíbrio nulo de (4.15) é assintoticamente estável.

\section{Exemplo 5:}

Seja

$$
x^{\prime}(t)=-b \operatorname{senh}(x(t))+a \int_{t-r}^{t} \operatorname{senh}(x(\tau)) d \tau ; \quad a r<b
$$

Neste exemplo, a função $g$ de (4.10) é dada por $g(x)=\operatorname{senh} x$. Portanto o equilíbrio nulo de (4.16) é assintoticamente estável.

Vamos, a seguir, estudar a estabilidade e a estabilidade assintótica do equilíbrio nulo de equaçôes da forma:

$$
x^{\prime}(t)=-a \int_{t-r}^{t} g(x(\tau)) d \tau, \quad t \geq 0
$$

onde $a$ e $r$ são constantes positivas. Para esta equação, podemos demonstrar o seguinte teorema:

Teorema 4.3: Seja $g$ uma função de classe $C^{\mathbf{1}}$ numa vizinhança da origem, satisfazendo $x g(x)>0$ para $x \neq 0$. Nessas condições, se $0<a r^{2} g^{\prime}(0) \leq 2$, o equilíbrio nulo de (4.17) é estável. Ainda mais, se $0<a r^{2} g^{\prime}(0)<2$, o equilíbrio nulo é assintoticamente estável. 
Demonstração: As condições $x g(x)>0$ para $x \neq 0$ e $g$ contínua implicam que $g(x)=0$ se, e somente se, $x=0$.

Mostremos inicialmente a estabilidade.

Seja $V(x)=x^{2} / 2$. Então $V^{\prime}\left(x_{t}\right)=x(t) x^{\prime}(t)=-a x(t) \int_{t-r}^{t} g(x(\tau)) d \tau$.

Suponhamos $V^{\prime}\left(x_{t_{1}}\right)>0$. Segue que

$$
-a x\left(t_{1}\right) \int_{t_{1}-\tau}^{t_{1}} g(x(\tau)) d \tau>0
$$

Consideremos $x\left(t_{1}\right)>0$. Logo $\int_{t_{1}-r}^{t_{1}} g(x(\tau)) d \tau<0$. Assim $g(x(t))$ muda de sinal em $\left[t_{1}-r, t_{1}\right]$. Logo, existe um $t_{2} \in\left[t_{1}-r, t_{1}\right)$ tal que $g\left(x\left(t_{2}\right)\right)=0$. Como $g(x)=0$ se, e somente se, $x=0$, segue que $x\left(t_{2}\right)=0$.

Pode ocorrer que exista mais de um $t$ com tal propriedade. Neste caso, tomamos $t_{2}$ tal que $x\left(t_{2}\right)=0$ e $x(t)>0$ para $t_{2}<t \leq t_{1}$.

Seja

$$
\delta_{0}=\inf \left\{g(x(t)): t \in\left[t_{1}-2 r, t_{1}\right]\right\}
$$

$\delta_{0}=g\left(x\left(t_{3}\right)\right), t_{3} \in\left[t_{1}-2 r, t_{1}\right], \delta_{0}<0$. Para $t \in\left[t_{1}-r, t_{1}\right]$, podemos escrever:

$$
x^{\prime}(t)=-a \int_{t-\tau}^{t} g(x(\tau)) d \tau \leq-\operatorname{ar} \delta_{0}=-\arg \left(x\left(t_{3}\right)\right)
$$

Seja $r_{1}=t_{2}-\left(t_{1}-r\right)=t_{2}-t_{1}+r$ e $0 \leq \tau<r-r_{1}$.

Temos que: 


$$
x^{\prime}(\tau) \leq-a g\left(x\left(t_{3}\right)\right)(r-\tau), \quad 0 \leq \tau<r-r_{1}
$$

Fixemos $x_{t_{1}-r+r_{1}}$ como condição inicial tal que $x(.)_{t_{1}-r+r_{1}}(\tau)=x\left(t_{1}-r+r_{1}+\tau\right)=x(\tau)$, $0 \leq \tau<r-r_{1}$. Basta observar que $t_{1}-r+r_{1}=t_{2}$.

Logo,

$\int_{0}^{r-r_{1}} x^{\prime}(\tau) d \tau \leq-a \int_{0}^{r-r_{1}} g\left(x\left(t_{3}\right)\right)(r-\tau) d \tau \leq-a g\left(x\left(t_{3}\right)\right) r \int_{0}^{r-r_{1}} d \tau+a g\left(x\left(t_{3}\right)\right) \int_{0}^{r-r_{1}} \tau d \tau$

$$
x\left(t_{1}\right)-x(0) \leq-a g\left(x\left(t_{3}\right)\right) r\left(r-r_{1}\right)+a g\left(x\left(t_{3}\right)\right)\left(r-r_{1}\right)^{2} / 2
$$

$$
x\left(t_{1}\right) \leq-a g\left(x\left(t_{3}\right)\right) r^{2} / 2+a g\left(x\left(t_{3}\right)\right) r_{1}^{2} / 2 .
$$

Assim,

$$
x\left(t_{1}\right) \leq-a g\left(x\left(t_{3}\right) r^{2} / 2 .\right.
$$

O Teorema do Valor Médio permite-nos escrever:

$$
g(0)-g\left(x\left(t_{3}\right)\right)=g^{\prime}\left(x^{*}\right)\left(0-x\left(t_{3}\right)\right), \quad x^{*} \in\left(x\left(t_{3}\right), 0\right),
$$

ou seja, 


$$
-g\left(x\left(t_{3}\right)\right)=-g^{\prime}\left(x^{*}\right) x\left(t_{3}\right)
$$

Substituindo em (4.18), vem que

$$
2 x\left(t_{1}\right) \leq a r^{2} g^{\prime}\left(x^{*}\right)\left(-x\left(t_{3}\right)\right)
$$

Como $0<a r^{2} g^{\prime}(0) \leq 2$ e $g \in C^{1}$, segue que $0<a r^{2} g^{\prime}\left(x^{*}\right) \leq 2$ numa vizinhança da origem. Logo,

$$
x^{2}\left(t_{1}\right) / 2 \leq x^{2}\left(t_{3}\right) / 2, \quad t_{3} \in\left[t_{1}-2 r, t_{1}\right]
$$

ou seja, $V\left(x\left(t_{1}\right)\right) \leq V\left(x\left(t_{3}\right)\right), t_{3} \in\left[t_{1}-2 r, t_{1}\right]$.

Para o caso em que $x\left(t_{1}\right)<0$, a técnica de demonstração é semelhante.

Para $V^{\prime}\left(x_{t_{1}}\right)=0$, segue que $x\left(t_{1}\right)=0$ ou $\int_{t_{1}-r}^{t_{1}} g(x(\tau)) d \tau=0$. Se $x\left(t_{1}\right)=0$, então para algum $t \in\left(t_{1}, t_{1}+r\right]$, devemos ter $x(t) \neq 0$, pois caso contrário, $x(t)=0$ para $t \in\left[t_{1}, t_{1}+r\right]$ e por unicidade $x(t)=0$, para $t \geq t_{1}$. Assim, existe um $t_{4} \in\left(t_{1}, t_{1}+r\right]$ tal que $x\left(t_{4}\right) \neq 0, V\left(x\left(t_{4}\right)\right)>0$ e $V^{\prime}\left(x_{t_{4}}\right)>0$. Sendo $x\left(t_{4}\right)>0$ ou $x\left(t_{4}\right)<0$ a análise é semelhante à feita no caso $V^{\prime}\left(x_{t_{1}}\right)>0$ e $x\left(t_{1}\right)>0$. Por outro lado, se $\int_{t_{1}-r}^{t_{1}} g(x(\tau)) d \tau=0$, segue que $g(x(t))$ muda de sinal em $\left[t_{1}-r, t_{1}\right]$. Logo existe um $t_{2} \in\left[t_{1}-r, t_{1}\right]$ tal que $g\left(x\left(t_{2}\right)\right)=0$ e o procedimento recai no caso $x\left(t_{2}\right)=0$, pois $g(x)=0$ somente para $x=0$.

Assim, temos: sempre que $V^{\prime}\left(x_{t_{1}}\right) \geq 0$, existe um $t_{3} \in\left[t_{1}-2 r, t_{1}\right]$ tal 
que $V\left(x\left(t_{1}\right)\right)-V\left(x\left(t_{3}\right)\right) \leq 0$. Portanto $V$ é dicotômica com relação à equação (4.17) e o Teorema (2.1) garante-nos a estabilidade do equilíbrio nulo de (4.17).

Passemos agora à estabilidade assintótica.

Da equação (4.19) temos:

$$
\frac{2 x\left(t_{1}\right)}{a r^{2} g^{\prime}\left(x^{*}\right)}<-x\left(t_{3}\right)
$$

Logo, $\left(2 / a r^{2} g^{\prime}\left(x^{*}\right)\right)^{2}\left(x^{2}\left(t_{1}\right) / 2\right)<x^{2}\left(t_{3}\right) / 2$.

Seja $p(y)=\left(2 / a r^{2} g^{\prime}\left(x^{*}\right)\right)^{2} y, y>0$, onde $2 / a r^{2} g^{\prime}\left(x^{*}\right)>1$ numa vizinhança da origem pois $0<a r^{2} g^{\prime}(0)<2$ e $g \in C^{1}$. Assim $p\left(V\left(x\left(t_{1}\right)\right)\right)<V\left(x\left(t_{3}\right)\right), t_{3} \in\left[t_{1}-2 r, t_{1}\right]$.

Para $x\left(t_{1}\right)<0$, o raciocinio é análogo.

Portanto, quando $V^{\prime}\left(x_{t_{1}}\right) \geq 0$ obtém-se $p\left(V\left(x\left(t_{1}\right)\right)\right)-V\left(x\left(t_{3}\right)\right)<0$ para algum $t_{3} \in\left[t_{1}-2 r, t_{1}\right]$. Logo, $V$ é estritamente dicotômica com relação à equação (4.17), onde o $T$ da Definição (1.10) é dado por $T=r$. Aplicando o Teorema (2.4), o equilíbrio nulo de (4.17) é assintoticamente estável.

\section{Exemplo: A equação}

$$
x^{\prime}(t)=-a \int_{t-r}^{t}\left[x(\tau)+x^{3}(\tau)\right] d \tau
$$

abordada por Lakshmikantham e Leela em [18] é um caso particular de (4.17) com $g(x)=x+x^{3}$. Assim para $0<a r^{2}<2$ temos estabilidade assintótica da solução nula da equação acima. 


\section{Capítulo 5}

\section{ANÁLISE DA ESTABILIDADE DE EQUAÇÕES COM RETARDAMENTO VARIÁVEL COM O TEMPO}

Neste capítulo vamos fazer algumas aplicações do método das funções dicotômicas no estudo da estabilidade das soluções de uma classe de equações do tipo $x^{\prime}(t)=f(t, x(t-r(t)))$ onde $r(t)$ é uma função contínua de $t$ e não negativa.

Inicialmente, fazemos o estudo de uma equação com retardamento fixo, isto por dois motivos básicos: esta equação tem sido bastante estudada e além disso, a técnica de abordagem é similar à empregada nas equações com retardamento variável, porém mais simples. Portanto o estudo desta equação torna mais fácil o entendimento do caso com retardamento variável.

Carvalho e Cooke em [4] estudaram, meramente como ilustração do emprego do método das funções dicotômicas, a estabilidade da equação escalar

$$
x^{\prime}(t)=-b x(t-r), \quad t \geq 0
$$

onde $b>0$ é um parâmetro dado. Hale também já havia abordado esta equação em [13] usando o subconjunto $W_{1}^{\infty} \subset C$, que consiste das funções absolutamente contínuas que tem derivada essencialmente limitada em $[-r, 0]$ e um teorema do tipo Razumikhin.

A equação $x^{\prime}(t)=-b(t) x(t-r)$, que é uma generalização de (5.1), foi analisada por Burton e Hatvani em [3], onde hipóteses mais fortes sobre a função $b$ são exigidas. Usando o método das funções dicotômicas, conseguimos melhorar como se verá adiante, 
os resultados de Burton e Hatvani e consequentemente os resultados de Carvalho e Cooke.

A seguir, fazemos um estudo de uma equação com retardamento variável, porém limitado, e concluimos o capítulo analisando a estabilidade de uma equação com retardamento infinito.

Em [3], a equação escalar

$$
\begin{aligned}
x^{\prime}(t) & =-b(t) x(t-r), \quad t \geq t_{0} \\
x_{t_{0}} & =\varphi
\end{aligned}
$$

foi estudada usando o Método Direto de Liapunov com funcionais sofisticados definidos em $C=C([-r, 0], R)$.

Considerando $b$ contínua, $b([0, \infty)) \subset[0,1]$ e impondo, além disso, as seguintes condições:

(i) $\quad-2+\int_{t-r}^{t}|b(u)| d u+r \leq 0$

(ii) $\quad b(t+r)=b(t), \quad \int_{t-r}^{t}|b(u)| d u>0$

(iii) $\int_{t-r}^{t}[1-|b(u)|] d u>0$,

foi mostrado, em [3], que para $\varphi \in C$ qualquer, as soluções $x\left(t, t_{0}, \varphi\right)$ de (5.2) tendem a zero quando $t$ tende a infinito.

Nós conseguimos através da teoria das funções dicotômicas, mostrar a estabilidade assintótica da solução nula de (5.2) sem a exigência das hipóteses (i), (ii), (iii), o 
que amplia significativamente a classe das funções $b$ para as quais estes resultados são válidos. Também a região de estabilidade em termos dos parâmetros $B$ e $r$ onde $B=\sup \{b(t): t \geq 0\}$, foi aumentada.

Nesse sentido podemos enunciar o seguinte teorema:

Teorema 5.1: Seja $b:[0, \infty) \rightarrow(0, \infty)$ uma função contínua e $B$ definido como anteriormente. Então se $0<B r<3 / 2$, a solução nula de (5.2) é assintoticamente estável. Ainda mais, se existir um $\varepsilon_{0}>0$ tal que $\lim _{t \rightarrow \infty} b(t)>\varepsilon_{0}$, a solução nula é um atrator global.

Demonstração: Uma vez mais usamos " $x(t)$ " para denotar a solução $x\left(t, t_{0}, \varphi\right)$.

Seja $V(x)=x^{2} / 2$. Mostremos que $V$ assim definida é estritamente dicotômica com relação à equação (5.2), e pelo Teorema (2.4) concluimos o resultado desejado.

Seja $t_{1}>0$ tal que $V^{\prime}\left(x_{t_{1}}\right) \geq 0$. Então $V^{\prime}\left(x_{t_{1}}\right)>0$ ou $V^{\prime}\left(x_{t_{1}}\right)=0$. Se $V^{\prime}\left(x_{t_{1}}\right)>0$, segue que $x\left(t_{1}\right) x^{\prime}\left(t_{1}\right)>0$.

Suponhamos $x\left(t_{1}\right)>0$. Então $x^{\prime}\left(t_{1}\right)>0$ e assim, $x(t)$ é crescente numa vizinhança de $t_{1}$.

Seja $t_{2}>t_{1} \circ$ primeiro ponto de máximo de $x(t)$, à direita de $t_{1}$. É fácil ver que existe $t_{2}$ com tal propriedade no intervalo $\left(t_{1}, t_{1}+r\right]$, o qual também é ponto de máximo de $V(x(t))$. Desta forma segue que $x\left(t_{2}-r\right)=0$.

Vamos dividir a demonstração em dois casos: $0<B r<1$ e $1 \leq B r<3 / 2$.

19. caso: $1 \leq B r<3 / 2$

Seja $x\left(t_{3}\right)=\inf \left\{x(t): t \in\left[t_{2}-3 r, t_{2}-r\right]\right\}$. Note que $x\left(t_{3}\right)<0$.

Para $t \in\left[t_{2}-2 r, t_{2}-r\right], \quad x^{\prime}(t)=-b(t) x(t-r) \leq-B x\left(t_{3}\right)$.

Assim, podemos escrever que 


$$
x\left(t_{2}\right)-x\left(t_{2}-r\right)=\int_{t_{2}-\tau}^{t_{2}} x^{\prime}(\tau) d \tau \leq-B \int_{t_{2}-r}^{t_{2}} x(\tau-r) d \tau
$$

A limitação de $x(t)$ no intervalo $\left[t_{2}-3 r, t_{2}-r\right]$ implica que $x^{\prime}(t)$ também é limitada em $\left[t_{2}-2 r, t_{2}-r\right]$ e a integral $\int_{t_{2}-r}^{t_{2}} x(\tau-r) d \tau$ pode ser majorada pela soma das áreas de um retângulo e de um triângulo, como mostra a Figura (4), ou seja,

$$
x\left(t_{2}\right)<B\left[\left(r-\frac{1}{B}\right) x\left(t_{3}\right)+\frac{1}{2 B} x\left(t_{3}\right)\right]
$$

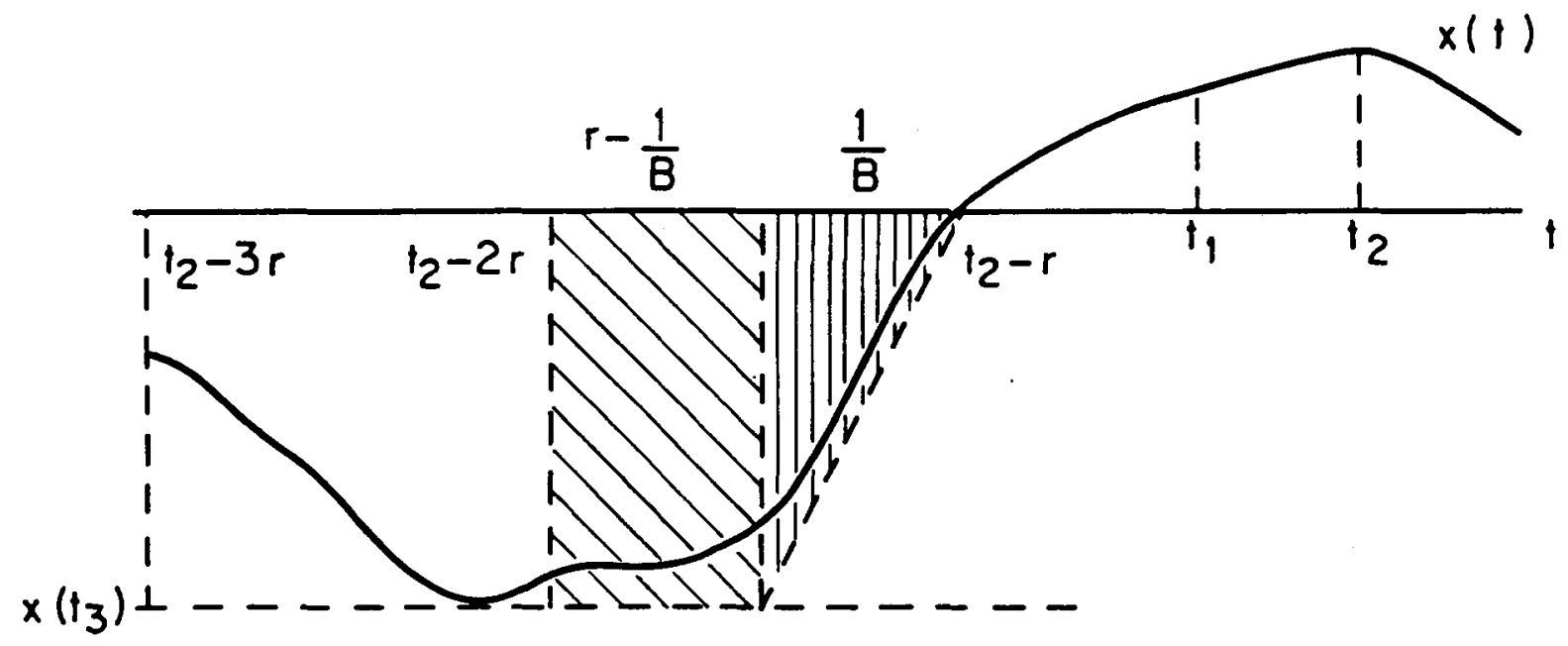

Figura 4

Assim, $x\left(t_{2}\right)<x\left(t_{3}\right)(B r-(1 / 2))$. Logo,

$$
\frac{x^{2}\left(t_{3}\right)}{2}>\frac{x^{2}\left(t_{2}\right)}{2(B r-(1 / 2))^{2}} .
$$


Como $1 \leq B r<3 / 2$, segue que $(B r-(1 / 2))<1$.

Definindo $p(y)=y /(B r-(1 / 2))^{2}, \quad y>0$, obtemos $V\left(x\left(t_{3}\right)\right)>p\left(V\left(x\left(t_{2}\right)\right)\right)$, para algum $t_{3} \in\left[t_{2}-3 r, t_{2}-r\right]$.

Considerando $x\left(t_{1}\right)<0$, a demonstração é semelhante.

Portanto para $x\left(t_{1}\right)>0$ ou $x\left(t_{1}\right)<0$ temos: sempre que $V^{\prime}\left(x_{t_{1}}\right)>0$, existem um $t_{2}>t_{1} \operatorname{com} V\left(x\left(t_{2}\right)\right)>V\left(x\left(t_{1}\right)\right)$ e um $t_{3}<t_{1}$ tal que $V\left(x\left(t_{3}\right)\right)>p\left(V\left(x\left(t_{2}\right)\right)\right)>$ $>p\left(V\left(x\left(t_{1}\right)\right)\right)$, isto é, quando $V^{\prime}\left(x_{t_{1}}\right)>0$, segue que $V\left(x\left(t_{3}\right)\right)>p\left(V\left(x\left(t_{1}\right)\right)\right)$, para algum $t_{3} \in\left[t_{2}-3 r, t_{2}-r\right]$. $\mathrm{O}$ valor de $T$, neste caso, é $2 r$.

29 caso: $0<B r<1$

Neste caso, como $r<1 / B$, o retângulo na Figura (4) deixa de existir e assim a integral $\int_{t_{2}-r}^{t_{2}} x(\tau-r) d \tau$ é limitada somente pela área do triângulo. Procedendo como no caso anterior obtemos $V\left(x\left(t_{3}\right)\right)>p\left(V\left(x\left(t_{2}\right)\right)\right)$, onde a função $p$ é dada por $p(y)=y /\left(B^{2} r^{2} / 2\right)^{2}, y>0$.

Vamos agora tratar o caso $V^{\prime}\left(x_{t_{1}}\right)=0$. Segue que $x\left(t_{1}\right)=0$ ou $x^{\prime}\left(t_{1}\right)=0$. Se $x\left(t_{1}\right)=0$, então para algum $t \in\left(t_{1}, t_{1}+r\right]$ devemos ter $x(t) \neq 0$, pois caso contrário, $x(t)=0$ para $t \in\left[t_{1}, t_{1}+r\right]$ e por unicidade $x(t)=0$, para $t \geq t_{1}$. Logo, se existir um $t_{4} \in\left(t_{1}, t_{1}+r\right]$ tal que $x\left(t_{4}\right) \neq 0$, segue que $V(x(t))$ terá um máximo nesse intervalo e assim repetiremos o raciocínio feito quando $x^{\prime}\left(t_{1}\right)>0$.

Se $x^{\prime}\left(t_{1}\right)=0$ com $x\left(t_{1}\right)>0$, então $t_{1}$ é ponto de máximo ou ponto de inflexão de $x(t)$ e consequentemente de $V(x(t))$. Em qualquer um dos casos procedemos como anteriormente. 
Portanto, $V$ é estritamente dicotômica com relação à (5.2).

Tomando $u(x)=x^{2} / 4$ e $v(x)=x^{2}, x>0$, a condição (2.1) está satisfeita e então pelo Teorema (2.4) a solução nula de (5.2) é assintoticamente estável.

Suponhamos, a seguir, que $\lim _{t \rightarrow \infty} b(t)>\varepsilon_{0}>0$ e mostremos que com essa condição adicional a solução nula é um atrator global.

De fato, para $\varphi$ qualquer, $\varphi \in C$, temos duas alternativas:

(a) existe um $\bar{t}$ tal que $x\left(t, t_{0}, \varphi\right)$ não tem raiz para $t \geq \bar{t}$ ou

(b) existe uma sequência $t_{n} \rightarrow \infty$ quando $n \rightarrow \infty$ com $x\left(t_{n}, t_{0}, \varphi\right)=0 \mathrm{e}$ $x\left(t, t_{0}, \varphi\right) \neq 0$ para $t_{n-1}<t<t_{n}$.

No caso (b), existe uma sequência $\left(t_{n}^{\prime}\right), t_{n-1}<t_{n-1}^{\prime}<t_{n}$, tal que $c_{n}=V\left(x\left(t_{n}^{\prime}, t_{0}, \varphi\right)\right)$ é um máximo local no intervalo $\left(t_{n-1}, t_{n}\right)$. Procedendo como na prova do Teorema (2.4) mostramos que $c_{n} \rightarrow 0$, quando $n \rightarrow \infty$.

No caso (a), podemos supor, sem perda de generalidade que $x\left(t, t_{0}, \varphi\right)>0$, para $t \geq \bar{t}$. Caso contrário, trabalhamos com $-x\left(t, t_{0}, \varphi\right)$. Isto implica que $x_{t}^{\prime}\left(., t_{0}, \varphi\right)<0$, para $t \geq \bar{t}$, isto é, $x\left(t, t_{0}, \varphi\right)$ é decrescente e limitada.

Seja $c=\lim _{t \rightarrow \infty} x\left(t, t_{0}, \varphi\right)$. Então $x\left(t-r, t_{0}, \varphi\right) \rightarrow c$ quando $t \rightarrow \infty$. Suponhamos $c>0$. Como $\lim _{t \rightarrow \infty} b(t)>\varepsilon_{0}>0$ para $t \rightarrow \infty$, existe um $t_{1}>\bar{t}$ tal que $b(t)>\varepsilon_{0} / 2$ para $t>t_{1}$. Podemos afirmar também que existe um $t_{2}>\bar{t}$ tal que $x\left(t-r, t_{0}, \varphi\right)>c / 2$ para $t>t_{2}$. Seja $\hat{t}=\max \left\{t_{1}, t_{2}\right\}$. Assim para $t \geq \hat{t}$, temos $b(t) x\left(t-r, t_{0}, \varphi\right)>\varepsilon_{0} c / 4 \mathrm{e}$ portanto $x\left(t, t_{0}, \varphi\right)<x\left(\hat{t}, t_{0}, \varphi\right)-(t-\hat{t}) \varepsilon_{0} c / 4$, o que implica em $\lim _{t \rightarrow \infty} x\left(t, t_{0}, \varphi\right)=-\infty$, o que é uma contradição. Logo $c=0$ e a solução nula é um atrator global de (5.2).

A seguir vamos abordar equações que apresentam retardamento variável, ou seja, $r=r(t)$.

Seja 


$$
\begin{aligned}
x^{\prime}(t) & =-b x(t-r(t)), \quad t \geq 0 \\
x_{0} & =\varphi
\end{aligned}
$$

onde $b>0, r(t)$ é contínuo, limitado e $r(t) \geq 0$.

Seja $c=\sup \{r(t): t \geq 0\}$.

Mediante essas observações vamos enunciar o teorema que garante a estabilidade assintótica do equilíbrio nulo de (5.3).

Teorema 5.2: Suponhamos $r(t), b$ e $c$ como anteriormente. Se $0<b c<3 / 2$, o equilíbrio nulo de (5.3) é assintoticamente estável.

Demonstração: Neste teorema também vamos dividir a demonstração em dois casos: $0<b c<1$ e $1 \leq b c<3 / 2$.

19. caso: $0<b c<1$

Para $t \geq c$, podemos escrever (5.3) como:

$$
\begin{aligned}
x^{\prime}(t) & =-b x(t-r(t))+b x(t)-b x(t) \\
& =-b x(t)+b \int_{t-r(t)}^{t} x^{\prime}(\tau) d \tau \\
& =-b x(t)-b \int_{t-r(t)}^{t} b x(\tau-r(\tau)) d \tau
\end{aligned}
$$


Seja $V(x)=x^{2} / 2$. Assim,

$$
V^{\prime}\left(x_{t}\right)=x(t) x^{\prime}(t)=-b x^{2}(t)-b x(t) \int_{t-r(t)}^{t} b x(\tau-r(\tau)) d \tau
$$

De acordo com a teoria de funções dicotômicas vamos procurar os pontos em que $V^{\prime}\left(x_{t}\right) \geq 0$.

Se $r(t)=0$, segue que $V^{\prime}\left(x_{t}\right)=-b x^{2}(t)$, ou seja, $V^{\prime}\left(x_{t}\right)<0$ se $x(t) \neq 0$, com o qual não precisamos nos preocupar.

Se $V^{\prime}\left(x_{t}\right) \geq 0$, então

$$
b\left(-x^{2}(t)-x(t) \int_{t-r(t)}^{t} b x(\tau-r(\tau)) d \tau\right) \geq 0 .
$$

A integral na desigualdade acima pode ser majorada da seguinte forma:

$$
\begin{aligned}
-x(t) \int_{t-r(t)}^{t} b x(\tau-r(\tau)) d \tau & \leq b|x(t)| \int_{t-\tau(t)}^{t}\left|x_{\tau-\tau(\tau)}(0)\right| d \tau \\
& \leq b r(t)|x(t)|\left\|x_{t-c}\right\| \\
& \leq b c|x(t)|\left\|x_{t-c}\right\|
\end{aligned}
$$

onde $\left\|x_{t-c}\right\|=\sup \{|x(t-c+\theta)|: \theta \in[-c, 0] \subset[-c, c)\}$ e $\left\|x_{t-c}\right\|=\left|x\left(t-c+\theta_{0}\right)\right|$, $\theta_{0} \in[-c, 0] \subset[-c, c)$, pois temos uma função contínua em compacto.

Logo, se $V^{\prime}\left(x_{t}\right) \geq 0$, usando a desigualdade anterior, podemos escrever:

$$
b\left(-x^{2}(t)+b c|x(t)|\left\|x_{t-c}\right\|\right) \geq b\left(-x^{2}(t)-x(t) \int_{t-r(t)}^{t} b x(\tau-r(\tau)) d \tau\right) \geq 0 .
$$


Assim, $b\left(-x^{2}(t)+b c|x(t)|\left\|x_{t-c}\right\|\right) \geq 0$, ou seja,

$$
b|x(t)|\left(-|x(t)|+b c\left\|x_{t-c}\right\|\right) \geq 0 .
$$

Segue que $\left\|x_{t-c}\right\| \geq|x(t)| / b c$.

Como $0<b c<1$, existe um $M, 0<b c<M<1$ tal que

$$
\left\|x_{t-c}\right\|>\frac{1}{M}|x(t)|
$$

Portanto,

$$
\frac{\left\|x_{t-c}\right\|^{2}}{2}>\frac{1}{M^{2}} \frac{|x(t)|^{2}}{2}
$$

Definindo $p(y)=y / M^{2}, y>0$, podemos afirmar: sempre que $V^{\prime}\left(x_{t}\right) \geq 0$, vem que $p(V(x(t)))-V(x(t-c+s))<0$ para algum $s \in[-c, c)$. Observe que de acordo com as notações usadas nas definições de dicotomia estrita, $s=\theta_{0}$ e $T=c$.

29 caso: $1 \leq b c<3 / 2$

Seja $t_{1}$ tal que $V^{\prime}\left(x_{t_{1}}\right) \geq 0$. Então $V^{\prime}\left(x_{t_{1}}\right)>0$ ou $V^{\prime}\left(x_{t_{1}}\right)=0$. Suponhamos $V^{\prime}\left(x_{t_{1}}\right)=x\left(t_{1}\right) x^{\prime}\left(t_{1}\right)>0$. Consideremos $x\left(t_{1}\right)>0$. Então $x^{\prime}\left(t_{1}\right)>0$ e assim $x(t)$ é 
crescente numa vizinhança de $t_{1}$. Logo, existe um $t_{2} \in\left(t_{1}, t_{1}+c\right]$ tal que $x^{\prime}\left(t_{2}\right)=0$, ou seja, $t_{2}$ é ponto de máximo de $x(t)$ e também de $V(x(t))$. Consequentemente $x^{\prime}\left(t_{2}\right)=-b x\left(t_{2}-r\left(t_{2}\right)\right)=0$, o que implica $x\left(t_{2}-r\left(t_{2}\right)\right)=0$. Observemos que necessariamente $r\left(t_{2}\right)>0$, pois caso contrário, $V^{\prime}\left(x_{t_{2}}\right)=-b x^{2}\left(t_{2}\right)<0$.

Seja $t_{3}=t_{2}-r\left(t_{2}\right)$. Chamemos $t_{4}=t_{3}-r\left(t_{3}\right)$.

Sabemos que $x\left(t_{4}\right)$ deve estar abaixo do eixo $x(t)=0$, pois caso contrário, a solução não cortaria o eixo em $t_{3}$.

Seja $r\left(t_{2}\right)=\varepsilon_{0}>0$. Pela continuidade de $r$ com relação a $t$, existe $\delta>0$ tal que se $t \in\left(t_{2}-\delta, t_{2}\right)$, então $r(t)>\varepsilon_{0} / 2>\delta$.

Sejam $t_{5}=t_{4}-r\left(t_{4}\right)=t_{3}-r\left(t_{3}\right)-r\left(t_{3}-r\left(t_{3}\right)\right)$ e $d=$ inf $\left\{x(t): t \in\left[t_{5}, t_{2}-\delta\right]\right\}$, $d=x(\bar{t}), \bar{t} \in\left[t_{5}, t_{2}-\delta\right]$. Note que $d<0$.

Para $t \in\left[t_{4}, t_{2}-\delta\right]$, temos

$$
x^{\prime}(t)=-b x(t-r(t)) \leq-b d
$$

Assim, podemos escrever:

$$
x\left(t_{2}\right)-x\left(t_{3}\right)=\int_{t_{3}}^{t_{2}} x^{\prime}(\tau) d \tau=-b \int_{t_{3}}^{t_{2}} x(\tau-r(\tau)) d \tau
$$

Esta última integral pode ser limitada pela soma das áreas de um retângulo e de um triângulo, e assim obtemos

$$
x\left(t_{2}\right)<b\left[d\left(c-\frac{1}{b}\right)+d \frac{1}{2 b}\right]
$$


Tal situação é semelhante à da equação (5.2), ilustrada na Figura (4), lembrando apenas que neste caso o retardo é variável.

Segue que

$$
x\left(t_{2}\right)<d\left(b c-\frac{1}{2}\right)
$$

ou seja,

$$
x\left(t_{2}\right)<x(\bar{t})\left(b c-\frac{1}{2}\right), \quad \bar{t} \in\left[t_{5}, t_{2}-\delta\right]
$$

Logo,

$$
\frac{x^{2}(\bar{t})}{2}>\frac{x^{2}\left(t_{2}\right)}{2(b c-(1 / 2))^{2}}
$$

Se $0 \leq b c<3 / 2$, então $(b c-(1 / 2))<1$.

Seja $p(y)=y /(b c-(1 / 2))^{2}, \quad y>0$.

Portanto, obtemos: sempre que $V^{\prime}\left(x_{t_{1}}\right)>0$, existe um $\bar{t} \in\left[t_{5}, t_{2}-\delta\right]$ tal que $V(x(\bar{t}))>p\left(V\left(x\left(t_{2}\right)\right)\right)>p\left(V\left(x\left(t_{1}\right)\right)\right)$.

Para $x\left(t_{1}\right)<0$ a demonstração é análoga e por isso vamos omití-la.

No caso em que $V^{\prime}\left(x_{t_{1}}\right)=0$, a técnica de demonstração é análoga àquela desenvolvida para a equação (5.2).

Observe que no caso $0<b c<1, T=c$ e no caso em que $1 \leq b c<3 / 2, T=2 c$. 
Portanto $V$ é estritamente dicotômica com relação à (5.3) e de acordo com o Teorema (2.4) o equilíbrio nulo de (5.3) é assintoticamente estável.

A seguir exibimos um exemplo de retardamento variável com as características citadas anteriormente.

Seja

$$
r(t)= \begin{cases}c(t-3 j), & t \in[3 j, 3 j+1] \\ -c t+(3 j+2) c, & t \in[3 j+1,3 j+2] \\ 0, & t \in[3 j+2,3 j+3]\end{cases}
$$

$j=0,1,2, \ldots$, e $c>0$. Observe que $r(t)$ é continuo, e satisfaz $0 \leq r(t) \leq c$. O gráfico de $r(t)$ é dado na Figura (5). Portanto o Teorema (5.2) assegura-nos que se $0<b c<3 / 2$, temos estabilidade assintótica do equilíbrio nulo de (5.3) com o retardamento $r(t)$ definido acima.

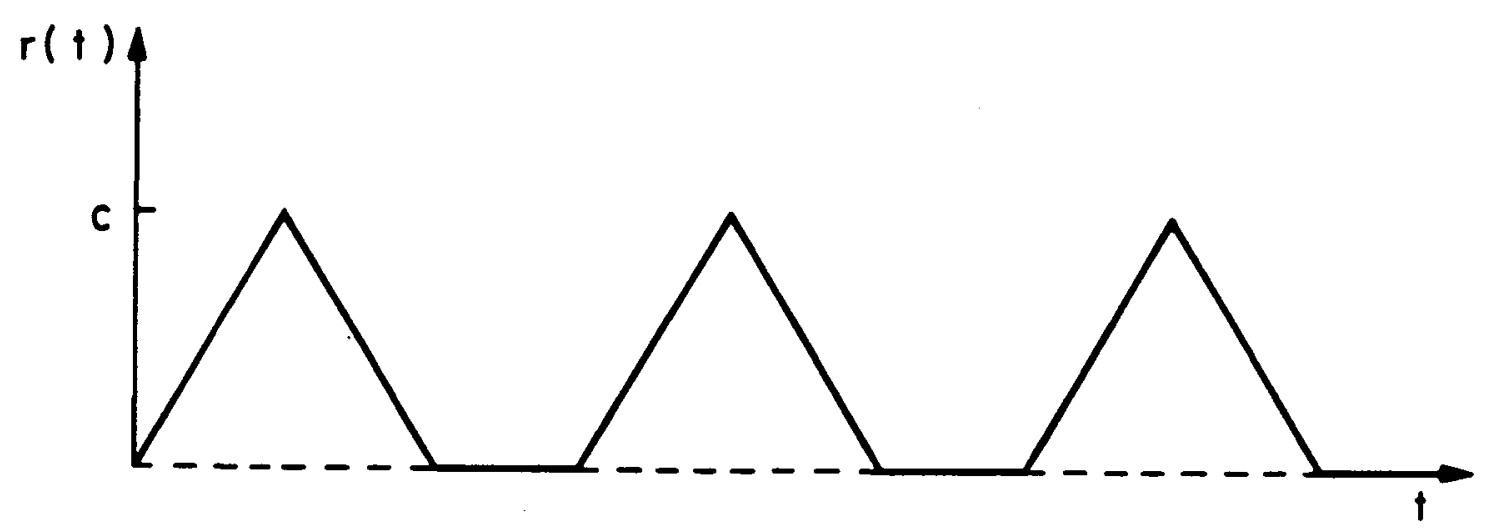

Figura 5 
É conveniente ressaltar aqui algumas características da equação (5.3) com relação às regiões de estabilidade em função do parâmetro $b$ e do retardo $r(t)$.

Mesmo no caso mais simples com retardamento $r(t)=r$ constante, estudos feitos através da equação característica associada à (5.3) mostram que ela não é estável para $b r>\pi / 2$ e é assintoticamente estável para $0<b r<\pi / 2$. Entretanto, no caso em que o retardo $r(t)$ é descontínuo isto pode não ser verdade como mostra o seguinte exemplo dado por Kolmanovskii e Nosov em [16].

Seja

$$
\begin{aligned}
x^{\prime}(t) & =-x(t-r(t)), \quad t \geq 0 \\
x_{0} & =\varphi(0) .
\end{aligned}
$$

Observe que $b=1$.

Seja $a \in(3 / 2, \pi / 2)$. Definimos o retardamento variável $\tilde{r}(t) \in[0, a]$ por

$$
\tilde{r}(t)= \begin{cases}t, & 0 \leq t \leq a \\ a, & a \leq t \leq a+1\end{cases}
$$

Para $t \geq a+1, \tilde{r}(t)$ é uma função periódica de periodo $a+1$, isto é, $\tilde{r}(t+(a+1))=\tilde{r}(t)$. Pelo método dos passos, obtemos para a solução de (5.4) com $r(t)=\tilde{r}(t)$ a expressão $x(k(a+1))=\varphi(0)((1 / 2)-a)^{k}, \quad k \in N$. Como $a>3 / 2$ temos $|(1 / 2)-a|>1$. Portanto $|x(k(a+1))| \rightarrow \infty$, quando $k \rightarrow \infty$. Assim a solução nula de (5.4) com este particular retardamento é instável. 
Para concluir, vamos analisar a estabilidade de uma equação com retardamento infinito.

Seja

$$
x^{\prime}(t)=-\frac{1}{t+1} x\left(t-\frac{t}{10}-1\right), \quad t \geq 0
$$

Neste caso $r(t)=((t / 10)+1)$.

Convém observar que o método de Razumikhin não é aplicável ao estudo da estabilidade de (5.5), uma vez que $r(t) \rightarrow \infty$, quando $t \rightarrow \infty$. Também um método de extensão proposto por Kato em [15] para o estudo de equações com retardamento infinito se mostra inadequado neste caso, pois a solução de (5.5) tende a zero de maneira oscilatória, característica essa que não satisfaz as hipóteses do método de Kato.

Através do método das funções dicotômicas vamos analisar a estabilidade de (5.5).

Seja $V(x)=x^{2} / 2$ e tomemos $t_{1}$ tal que $V^{\prime}\left(x_{t_{1}}\right) \geq 0$. Então $x\left(t_{1}\right) x^{\prime}\left(t_{1}\right) \geq 0$. Consideremos $x\left(t_{1}\right) \geq 0$. Assim, $x^{\prime}\left(t_{1}\right)=-x\left(t_{1}-\left(t_{1} / 10\right)-1\right) /\left(t_{1}+1\right) \geq 0$, ou seja, $x\left(t_{1}-\left(t_{1} / 10\right)-1\right) \leq 0$.

Podemos escrever:

$$
x\left(t_{1}\right)-x\left(t_{1}-\frac{t_{1}}{10}-1\right)=\int_{t_{1}-\left(t_{1} / 10\right)-1}^{t_{1}} x^{\prime}(\tau) d \tau=\int_{t_{1}-\left(t_{1} / 10\right)-1}^{t_{1}}-\frac{1}{\tau+1} x\left(\tau-\frac{\tau}{10}-1\right) d \tau .
$$

Como $x\left(t_{1}-\left(t_{1} / 10\right)-1\right) \leq 0$, segue que

$$
x\left(t_{1}\right) \leq \int_{t_{1}-\left(t_{1} / 10\right)-1}^{t_{1}}-\frac{1}{\tau+1} x\left(\tau-\frac{\tau}{10}-1\right) d \tau .
$$


No intervalo $\left[t_{1}-\left(t_{1} / 10\right)-1, t_{1}\right]$, o termo $1 /(\tau+1)$ é positivo e decrescente. Assim $1 /(\tau+1) \leq 10 / 9 t_{1}$.

Para $\tau \in\left[t_{1}-\left(t_{1} / 10\right)-1, t_{1}\right]$

$$
\left(\tau-\frac{\tau}{10}-1\right) \in\left[\left(t_{1}-\frac{t_{1}}{10}-1\right)-\left(\frac{t_{1}}{10}-\frac{t_{1}}{100}-\frac{1}{10}\right)-1, t_{1}-\frac{t_{1}}{10}-1\right]
$$

isto é, $(\tau-(\tau / 10)-1) \in\left[\left(81 t_{1}-190\right) / 100,\left(9 t_{1}-10\right) / 10\right]$.

Então, tomando $t_{2}$ tal que $x\left(t_{2}\right)$ seja o mínimo de $x(t)$ no intervalo $\left[\left(81 t_{1}-190\right) / 100,\left(9 t_{1}-10\right) / 10\right]$, podemos escrever

$$
x\left(t_{1}\right) \leq-\frac{10 x\left(t_{2}\right)}{9 t_{1}}\left[\frac{9 t_{1}-10}{10}-\frac{81 t_{1}-190}{100}\right]
$$

ou seja,

$$
x\left(t_{1}\right) \leq-x\left(t_{2}\right) \frac{\left(t_{1}+10\right)}{10 t_{1}}
$$

Logo, para $t_{1} \geq 10 / 9$, temos $x\left(t_{1}\right) \leq-x\left(t_{2}\right)$ e portanto $V\left(x\left(t_{1}\right)\right) \leq V\left(x\left(t_{2}\right)\right)$, para algum $t_{2} \in\left[\left(81 t_{1}-190\right) / 100,\left(9 t_{1}-10\right) / 10\right]$. Assim $V$ é dicotômica com relação à equação (5.5).

Escolhendo $u(x)=x^{2} / 4$ e $v(x)=x^{2}, x>0$ todas as condições do Teorema (2.1) estão satisfeitas e desta forma a solução nula de (5.5) é estável.

Observe, que neste caso, o $T\left(t_{1}\right)$ da Definição (1.9) é dado por 


$$
T\left(t_{1}\right)=\frac{t_{1}}{10}-\frac{t_{1}}{100}-\frac{1}{10}+1 .
$$

Para o caso $x\left(t_{1}\right) \leq 0$ faz-se uma análise similar. 


\section{BIBLIOGRAFIA}

[1] BENÁ, M. A. Estudo da estabilidade da equação $x^{\prime}(t)=-b(t) x(t-r)$ usando funções dicotômicas. In: Seminário Brasileiro de Análise, 36, São José dos Campos, 1992. Atas. São José dos Campos, SBA, 1992, p. $131-138$.

[2] BENÁ, M. A.; CARVAlHo, L. A. V. Some new results on stability of nonautonomous retarded functional differential equations using dichotomic maps. (submitted).

[3] BURTON, T.; HATVANI, L. Stability theorems for nonautonomous functional differential equations by Liapunov functionals. Tôhoku Math. J., v. 41, p. 65-104, 1989.

[4] CARValho, L. A. V.; COOKE, K. L. On dichotomic maps for a class of differential - difference equations. Proc. Royal Soc. Edinburgh, v. 117A, p. 317-328, 1991.

[5] CARVAlHO, L. A. V.; FERREIRA, R. R. On a new extension of Liapunov's direct method to discrete equations. Q. Appl. Math., v. 46, n. 4, p.779- 788, 1988.

[6] DOS REIS, J. G. Alguns aspectos da teoria de estabilidade para equações diferenciais funcionais do tipo neutro. São Carlos, ICMSC USP, 1976. (Tese de Doutorado).

[7] DOS REIS, J. G. Relações entre diferentes definições de estabilidade no sentido de Liapunov. São Carlos, ICMSC-USP, 1972. (Dissertação de Mestrado). 
[8] DRIVER, R. D. Ordinary and delay differential equations. New York, Springer - Verlag, 1977. (Appl. Math. Sciences, 20).

[9] HADDOCK, J. R.; KUANG, Y. Asymptotic theory for a class of nonautonomous delay differential equations. J. Math. Anal. Appl., v. 168, p. $147-162,1992$.

[10] HALANAY, A. Differential equations: stability, oscillations, time lags. New York, Academy Press, 1966.

[11] HALE, J. K. Asymptotic behavior of dissipative systems. Providence, AMS, 1988. (Math. Surveys and Monographs, 25).

[12] HALE, J. K. Functional differential equations. New York, SpringerVerlag, 1971. (Appl. Math. Sciences, 3).

[13] HALE, J. K. Theory of functional differential equations. New York, Springer - Verlag, 1977.

[14] IZÉ, A. F.; DOS REIS J. G. Contributions to stability of neutral functional differential equations. J. Differential Equations, v. 29, n. 1, p. $58-65,1978$.

[15] KATO, J. Stability problem in functional differential equations with infinite delay. Funkcial. Ekvac., v. 21, p. 63 - 80, 1978.

[16] KOLMANOVSKII, V. B.; NOSOV, V. R. Stability of functional differential equations. London, Academic Press, 1986.

[17] KRASOVSKII, N. N. Stability of motion. Stanford, Stanford University Press, 1963. 
[18] LAKSHMIKANTHAM, V.; LEELA, S. A technique in stability theory of delay - differential equations. Nonlinear Analysis, Theory, Methods $\mathcal{B}$ Applications, v. 3, n. 3, p. 317 - 323, 1979.

[19] LAKSHMIKANTHAM, V.; LEELA, S. Differential and integral inequalities, v. II. New York, Academic Press, 1969.

[20] LA SALLE, J.; LEFSCHETZ, S. $\quad$ Stability by Liapunov's direct method with applications. New York, Academic Press, 1961.

[21] MACKEY, M. C.; GLASS, L. Oscillation and chaos in physiological control systems. Science, v. 197, p. 287 - 289, 1977.

[22] MALLET-PARET, J.; NUSSBAUM, R. D. A differential-delay equation arising in Optics and Physiology. Siam J. Math. Anal., v. 20, n. 2, p. $249-292,1989$.

[23] MALLET-PARET, J.; NUSSBAUM, R. D. Global continuation and asymptotic behavior for periodic solutions of a differential delay equation. Ann. Math. Pura Appl., v. 145, p. 33 - 182, 1986.

[24] MAY, R. M. Nonlinear phenomena in Ecology and Epidemiology. Nonlinear dynamics. Annals of the New York Academy of Science, v. 357,1980 .

[25] WEN, L. Z. On the uniform asymptotic stability in functional differential equations. Proc. Amer. Math. Soc., v. 85, n. 4, p. $533-538,1982$. 